(Aus dem pharmakologischen Institut der Reichsuniversität Utrecht.)

\title{
Zur Regelung der Bewegungen durch das Zentralnervensystem.
}

III. Mitteilung.

Von

R. Magnus.

(Mit 5 Textfiguren.)

Inhaltsverzeichuis. Seite

1. Einleitung . . . . . . . . . . . . . . 545

2. Sind für das Zustandekommen der Schaltung zentripetale Nerven erforderlich? . . . . . . . . . . . . . . . 547

a) Versuche an Hunden. . . . . . . . . . . . . . . . . . . 548

b) Versuche an Katzen . . . . . . . . . . . . . . . . . . . 557

c) Zusammenfassung der Ergebnisse . . . . . . . . . . . . 567

3. Durch welche zentripetalen Nerven wird die Schaltung vermittelt? . . 567

4. Zusammenfassung . . . . . . . . . . . . . 572

5. Über den zentralen Sitz der Schaltung . . . . . . . . . 573

6. Die tonische Natur der Schaltung . . . . . . . . . . . 576

7. Die Rolle der Schaltung bei der Ausführung normaler Körperbewegungen 578

8. Schlusssätze . . . . . . . . . . . . . 583

\section{Einleitung.}

In zwei fruhheren Mitteilungen: „Zur Regelung der Bewegungen durch das Zentralnervensystem " habe ich über Versuche an Hunden ${ }^{1}$ ) und Katzen ${ }^{2}$ ) berichtet, in denen es möglich gewesen war, auf ein und denselben afferenten Reiz hin verschiedene und manchmal direkt entgegengesetzte Reflexbewegungen zu erhalten. Es hatte sich dabei ergeben, dass man den Reizerfolg nach Willkür beherrschen kann

1) R. Magnus, Zur Regelung der Bewegungen durch das Zentralnervensystem. I. Mitt. Pflüger's Arch. Bd. 130 S. 219. 1909.

2) R. Magnus, Zur Regelung der Bewegungen durch das Zentralnervensystem. II. Mitt. Pflüger's Arch. Bd. 130 S. 253. 1909. 
durch die Lage und Stellung, welche man dem Gliede vorher gibt, an welchem der Reflex beobachtet werden soll. An dem Hinterbein des Räckenmarkshundes erfolgt auf die verschiedenartigsten Reize (am besten von der kontralateralen Seite) bei gebeugtem Beine Streckung, bei gestrecktem Beine Beugung, nach Abduktion Adduktion und umgekehrt. Der Schwanz der Rückenmarkskatze schlägt auf Berührung der äussersten Spitze regellos nach allen Seiten, wenn das Organ sich vorher in symmetrischer Lage zur Körperachse befand; wird aber der Schwanz vorher nach irgendeiner Seite abgelenkt, so erfolgt nunmehr auf denselben Reiz ganz gesetzmässig die Bewegung nach der entgegengesetzten, d. h. der gedehnten Seite. Es wurde ferner auseinandergesetzt, dass dieses Resultat in beiden Fällen nicht auf der anatomischen Anordnung der Skeletteile und der Muskulatur beruhen kann (wofür in der vorliegenden Mitteilung noch weitere zwingende Beweise gebracht werden sollen), sondern anf "Schaltungen" im Zentralnervensystem bezogen werden muss. Für diese Schaltungen gilt in den bisher studierten Fällen die von v. Uexküll ${ }^{1}$ ) bei Wirbellosen aufgestellte Regel, dass in einem diffusen Zentralorgan die Erregung immer am leichtesten den Zentren derjenigen Muskeln zufliesst, welche sich in Zustand der grössten Dehnung befinden. Es stellt sich also die Peripherie das Zentralorgan selbst ein, und je nach dem wechselnden Zustand der Peripherie ist die Verteilung der Erregbarkeiten und Schaltungen im Zentrum eine andere. Die zweite Mitteilung schloss mit den Worten:

„In welcher Weise nun es zustande kommt, dass durch die veränderte Lage des Gliedes eine veränderte Schaltung im Rückenmarke eintritt, kann aus den bisherigen Versuchen nicht abgeleitet werden. Die Frage, ob es wirklich die Dehnung der Muskeln ist, oder ob es die Gelenke sind, oder, was unwahrscheinlicher wäre, ob es die Haut ist, von der aus die Reaktion ausgelöst wird, und welche Nervenbahnen für diese Beeinflussung des Zentrums von der Peripherie her in Betracht kommen, inuss durch besondere Versuche entschieden werden."

Die Beantwortung dieser letzteren Fragen ist das Thema der vorliegenden Mitteilung.

1) J. v. Uexküll, Die ersten Ursachen des Rhythmus in der Tierreihe. Ergebn. d. Physiol. Bd. 3 (2) S. 1. 1904. 


\section{Sind für das Zustandekommen der Schaltung zentripetale Nerven erforderlich?}

Wenn man die Frage entscheiden will, auf welchem Wege das Zentralnervensystem "erfährt", dass irgendwelche Veränderungen in der Lage und Stellung der Glieder vorgenommen sind, so kann an zwei Möglichkeiten gedacht werden. Entweder es handelt sich um Reflexe. Durch die veränderte Lage und Stellung des Gliedes werden in der Haut, den Gelenken, den Sehnen oder den Muskeln sensible Nervenendigungen erregt, und von hier aus dem Zentralnervensystem durch die hinteren Wurzeln Impulse zugeleitet, welche dort eine Änderung der Erregbarkeitsverteilung bewirken, mit dem Resultat, dass nun ein nachfolgender Reiz die Zentren anderer Muskeln in Erregung versetzen kann als vorher.

Diese Annahme ist zwar weitans die wahrscheinlichste, aber ist keineswegs die einzig mögliche. Man kann sich auch vorstellen, dass die Erregbarkeitsverteilung von den Muskeln aus beeinflusst wird auf dem Wege dureh die zentrifugalen motorischen Nerven. Ob man nämlich ein Gegner oder eip Anhänger der Neuronenlehre ist, so wird man doch wenigstens zugeben müssen, dass motorisehes Zentrum (Vorderhornzelle), motorischer Nerv und Skelettmuskel ein funktionell zusammengehöriges Ganze bilden, und es wäre immerhin vorstellbar, dass durch Zustandsänderungen in der Peripherie (in Muskel) die Erregbarkeit des Zentrums beeinflusst werden könnte. Ähnliche Vorstellungen sind durch v. Uexkü $11^{1}$ ) zur Erklärung der von ihm bei Wirbellosen beobachteten Schaltungen entwickelt worden. Ihre Übertragung auf das Wirbeltier schien mir im Beginn dieser Untersuchungsreihe keineswegs eine Unmöglichkeit zu sein. Wissen wir doch, dass nach Fortfall zentripetaler Impulse noch rhythmische alternierende Bewegungen möglich sind, dass ein Hund mit einer asensiblen Pfote noch laufen, ein Tabiker noch gehen kann. Es war eben zu untersuchen, ob bei diesen Bewegungen asensibler Extremitäten sich noch solche Schaltungen und Umkehrphänomene nachweisen lassen, wie sie in den vorhergegangenen Mitteilungen beschrieben worden sind. Der Beantwortung dieser Frage dienen die in der ersten Hälfte der vorliegenden Arbeit geschilderten Ver-

1) J. v. Uexküll, Studien über den Tonus. II. Die Bewegungen der Schlangensterne. Zeitschr. f. Biol. Bd. 46 S. 1. 1904. 
suche. Das Ergebnis derselben ist, dass nach Durchtrennung der zentripetalen Bahnen die untersuchten Schaltungen und Umkehrreaktionen sich nicht mehr beobachten lassen.

a) Versuche an Hunden.

Die Versuche an Hunden bezwecken festzustellen, ob die in der ersten Mitteilung ${ }^{1}$ ) geschilderten Schaltungen nach Ausschluss der hinteren Wurzeln noch vorhanden sind. Es handelte sich um Tiere, denen das Rückenmark im unteren Thorakalteile durchtrennt war. Als reflexauslösende Bahnen konnten die Fasern für die oberflächliche und tiefe Hautsensibilität sowie für die Sehnen-, Periost- und Muskelempfindlichkeit benutzt werden. Der Reizerfolg wurde am kontralateralen Hinterbein beobachtet, und an diesem letzteren wurde auch durch die veränderte Stellung, die man dem Gliede gab, die Schaltung im Zentralnervensystem bewirkt. Dieses Glied, das „Erfolgsbein" also, musste asensibel gemacht werden, und zwar so vollständig als möglich. Danach ergab sich folgender Versuchsplan: Einem gesunden Hunde werden zunächst intradural, um jede Möglichkeit einer Nervenregeneration auszuschliessen, die Hinterwurzeln der einen Seite im ganzen Lumbal- und Sakralmark durchtrennt. Die Wunde wird zur Heilung gebracht und abgewartet, bis das asensible Bein wieder in der bekannten "ataktischen" Weise zum Gehen benutzt wird. Wenn die Motilität des Beines sich völlig wiederhergestellt hat und die motorischen Apparate des Lendenund Sakralmarks wieder in guter Weise funktionieren, wird die Sensibilität auf der operierten Seite sorgfältig bestimmt. Erst wenn sich ergibt, dass die operierte Pfote völlig asensibel ist und bleibt, wird die Querdurchtrennung des Rückenmarks im unteren Brustteile vorgenommen. Dann wird wieder gewartet, bis die Wunde geheilt ist und bis alle Shokerscheinungen zurückgegangen sind. Wenn die asensible Pfote danach wieder gute Motilität zeigt, können nunmehr von der sensiblen Pfote gekreuzte Reflexe auf die asensible ausgelöst werden, und man kann untersuchen, ob sich durch veränderte Lage und Stellung der asensiblen Pfote noch die früher beschriebenen Schaltungsphänomene erzielen lassen. Nur wenn man in dieser allerdings langwierigen und etwas mühseligen Weise vorgeht, ist

1) R. Magnus, Zur Regelung der Bewegungen durch das Zentralnervensystem. I. Mitt. Pflüger's Arch. Bd. 130 s. 219. 1909. 
Zur Regelung der Bewegungen durch das Zentralnervensystem. III. 549

man wirklich sicher, ein Objekt von guter Motilität und mit genau bekannter Sensibilitätsstörung zu haben. Diesen Anforderungen genügen zwei Hunde, die ich im letzten Jahre operiert habe.

\section{Versuch 1.}

"Gerda", weibl. junger Foxterrier.

27. November 1909. In Ätherchloroformnarkose wird der Wirbelkanal vom 13. Brustwirbel an bis weit ins Kreuzbein hinein eröffnet, die Dura der Länge nach gespalten und die hinteren Wurzeln vom Unterrande der letzten Rippe bis zum Ende des Rückenmarkes auf der rechten Seite durchschnitten. Dauer der Operation 1 Stunde. 1/2 Stunde später lässt sich links, nicht aber rechts der Patellarreflex auslösen.

4. Dezember. Bei Schlag auf die linke Kniesehne Bewegung des rechten Beines (erstes Auftreten gekreuzter Reflexe).

13. Dezember. Wunde geheilt. Wird der Hund frei hängend in der Luft gehalten, so führen beide Beine Spontanbewegungen aus.

17. Dezember. Beginnt auf vier Pfoten zu stehen und zu laufen.

28. Dezember. Erste kinematographische Aufnahme von Stehen und Laufen. Das Tier steht auf vier Pfoten, das rechte asensible Bein hat deutlichen Tonus, wenn auch weniger als das linke. Gelegentlich lassen sich spontane Beugebewegungen der rechten Pfote beobachten. Beim Laufen wird das rechte Bein meist auf dem Fussrücken nachgeschleift, führt aber gelegentlich einen Schritt aus, bei dem dann starkes Ausfahren nach der linken Seite auftritt. In der Folgezeit entwickelt sich nun das bekannte Bild der Bewegungsstörung asensibler Extremitäten, wie es durch Baldi ${ }^{1}$ ), Hering ${ }^{2}$, Muskens ${ }^{3}$,

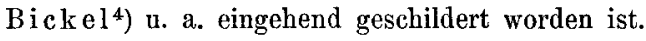

24. Januar 1910. Der Hund verwundet sich seine asensible Pfote selbst. Die Heilung dauert bis zum 16. Februar.

19. Februar. Zweite kinematographische Aufuahme. Der Hund läuft abwechselnd auf drei und anf vier Pfoten, Schritt, Trab und Galopp. Dabei wird das Bein entweder auf dem Fussrücken nachgeschleift, meist macht es aber die Laufhewegungen mit. Diese werden mit Kraft ausgeführt, zeichnen sich durch Schnelligkeit und Exkursionsgrösse aus und haben einen ausfahrenden Charakter. Häufig ist eine abnorme Richtung der Bewegungen nach innen oder aussen zu bemerken. Treppensteigen möglich. Beim Stehen wird der Fuss oft durch plötzliche Beugebewegungen des rechten Beines vom Boden abgehoben. Steht

1) D. Baldi, Effetti della recisione delle radici posteriori sui movimenti. Lo sperimentale 1885 p. 265 . (Zit. nach Bickel.)

2) H. E. Hering, Über Bewegungsstörungen nach zentripetaler Lähmung. Schmiedeberg's Arch. Bd. 38 S. 266.1897.

3) L. J. J. Muskens, Demonstration of exper. ataxia and its recovery. Journ. Boston Soc. med. scienc. vol. 11. 1898.

4) A. Bickel, Über den Einfluss der sensiblen Nerven und der Labyrinthe uf die Bewegungen der Tiere. Pflüger's Arch. Bd.67 S. 299. 1897. 
längere Zeit auf dem rechten Fussrücken oder lässt den Fuss über den Tischrand herabhängen, ohne die Stellung zu korrigieren. Der Tonus des rechten Beines ist hinreichend, um beim Gehen das Gewicht des Hinterkörpers allein zu tragen, aber deutlich geringer als links. Bei Schlag auf die linke Kniesehne erfolgt Beugung des rechten Beines in jeder Stellung. - Resümee: Die Motilität des asensiblen Beines hat sich nach der Operation wieder völlig hergestellt.

23. Februar. Bestimmung der Sensibilitätsgrenze des asensiblen Beines, deren Ergebnis mit drei früheren Bestimmungen am 19. Dezember, am 24. Dezember und am 17. Januar genau übereinstimmt. Die Grenze lässt sich besonders gut bestimmen, weil der Hund sehr scharf, besonders auf Kneifen mit einer
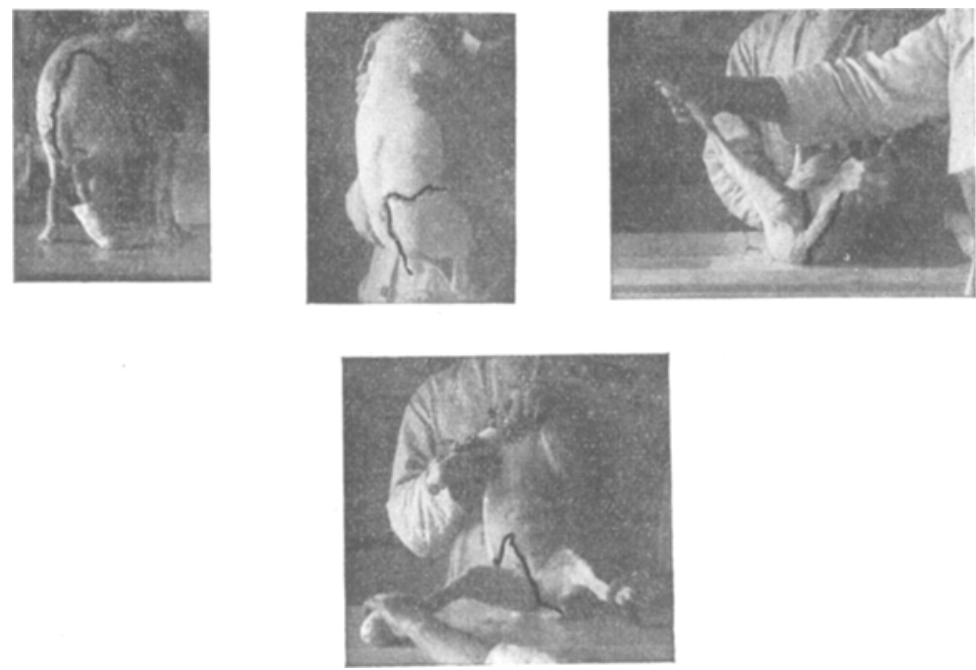

Fig. 1.

Pinzette reagiert. Zwischen je einer sensiblen und asensiblen Hautstelle wird auf dem weissen Fell mit schwarzer Farbe eine Linie gezogen und darauf das Tier photographiert. (Fig. 1). Das ganze rechte Hinterbein ist asensibel. Trotz mehrfacher Prüfung lassen sich auch keine kleinen sensiblen Inseln entdecken. Von dem Bein greift die gefühllose Hautpartie anf den Rumpf über, wo sie ebenfalls ein zusammenhängendes Ganze ohne Inseln bildet. Die Grenze verläuft von der Schwanzspitze genau medial an der Ventralseite des Schwanzes gegen den After, verläuft am Damm genau medial über die Vulva, dann am Baucie zunächst genau in der Linea alba, um zwischen letzter und vorletzter Mamilla etwas nach rechts auszubiegen und $1-1^{1 / 2} \mathrm{~cm}$ neben der Linea alba herzulaufen. Einen Fingerbreit oberhalb der vorletzten Mamilla (etwas unterhalb des Nabels) biegt die Grenze dann scharf lateral um und verläuft um den ganzen Rumpf ziemlich genau in einer frontalen Ebene bis gegen die Wirbelsäule. Die bei gestrecktem Hinterbein sich sehr scharf von der seitlichen Bauchwand abhebende Schenkelfalte wird von der Sensibilitätsgrenze etwa 1-2 Finger breit hinter ihrem 
kranialen Ansatze geschnitten. Am Rücken verläuft die Grenze dann wieder genau medial der Wirbelsäule folgend bis zur Schwanzspitze. Diese Sensibilitätsgrenze hat sich bei wiederholter Prüfung bis heute (2. Juli 1910) nicht geändert.

Es erhebt sich nun die Frage, ob der gefundene Sensibilitätsausfall übereinstimmt mit der beabsichtigten Operation, der Durchschneidung aller lumbalen und sakralen Wurzeln. Über diesen Punkt bin ich in freundlichster Weise durch Prof. C. Winkler in Amsterdam aufgeklärt worden, der kürzlich gemeinsam mit G. van Rynberk die segmentale Verteilung der sensiblen Wurzeln in der Haut des Hundes untersucht hat ${ }^{1}$ ). Es kommen sehr zahlreiche Varietäten vor, die zum Teil mit Varietäten in der Zahl der Brust und Lendenwirbel zusammenhängen. Die daraufhin bei dem Hunde "Gerda" vorgenommene Röntgenuntersuchung ergab, dass derselbe 13 rippentragende und 7 lumbale Wirbel besitzt, also normaie Skelettrerhältnisse zeigt. Danach ist es nach Mitteilung von Prof. Winkler höchst wahrscheinlich, dass die von mir festgestellte Sensibilitätsgrenze die caudale Grenzlinie des 13 . Thorakalnerven ist, und dass somit die erste hintere Lendenwurzel noch mit durchschnitten worden ist; die 2. Lumbalwurzel sei dagegen sicher durchtrennt.

Über die segmentale motorische Innervation der Beinmuskeln durch die ersten vier Lendennerven beim Hunde liegen noch nicht sehr zahlreiche Untersuchungen vor; Sherrington ${ }^{2}$ ) gibt nach drei Versuchen an, dass die Verhältnisse ähnlich liegen wie bei der Katze. Nach seinen Ergebnissen würde die 4. vordere Lendenwurzel den Hauptteil der Innervation des Iliopsoas liefern, die 3. sich auch noch deutlich daran beteiligen und die 2. Lumbalwurzel noch leichte Beugung des Oberschenkels bewirken können. Die 1. Lendenwurzel beteiligt sich nach diesen Versuchen nicht mehr an der motorischen Innervation des Beines.

Aus dem Vorhergehenden folgt (wenn man berücksichtigt, dass die motorischen und sensiblen Nervenbahnen eines Muskels ausnahmslos (Sherrington) im gleichen Niveau das Rückenmark verlassen), dass durch die vorgenommene Hinterwurzeldurchschneidung sowohl die Haut als die Muskulatur des rechten Hinterbeines, soweit sie bei Bewegungen desselben in Mitleidenschaft gezogen werden können, vollständig asensibel gemacht worden sind.

3. März 1910. In Ätherchloroformnarkose wird der Rückenmarkskanal am 11. Brustwirbel eröffnet und das Rückenmark durchtrennt. Naht der Wunde in zwei Etagen. Nach 4 Stunden ist der Patellarreflex am linken Beine lebhaft.

5. März. Spontanbewegungen des rechten (asensiblen) Beines, aber noch keine gekreuzten Reflexe.

12. März. Gekreuzter Patellarreflex vom linken auf das rechte Bein auslösbar.

18. März. Gekreuzter Streckreflex von links nach rechts.

1) Anm. bei der Korrektur: Ein Teil dieser Untersuchungen ist inzwischen veröffentlicht: C. Winkler en G. A. van Rynberk, Exp. onderzoek. over segmenteelinnervatie van de huid van den hond. VI. - Sitzungsber. d. k. Akademie d. Wiss. in Amsterdam. Mat.-naturw. Abt. 25. Juni 1910.

2) C. S. Sherrington, Notes on the arrangement of some motor fibres in the lumbo-sacral plexus. Journ. of physiol. vol. 13 p. 621.1892. 
13. April. Erste Andeutung von Pendelbewegungen der Hinterbeine, wenn das Tier frei in der Luft gehalten wird. Dieses Pendeln ist vom 2. Mai ab deutlich und kräftig, wird durch Drücken des Schwanzes verstärkt und wird am 18. Mai und 22. Juni kinematographisch aufgenommen. Dabei ist zu bemerken, dass das asensible Bein des Rückenmarkshundes deutlich ataktische Bewegungen beim Pendeln ausführt. Die Bewegung beginnt mit einer kurzen Streckung des sensiblen linken Beines, daran schliesst sich schnell, noch ehe die Streckung ibr Maximum erreicht hat, eine sehr schnelle, ausgiebige und stark nach der Seite ausfahrende Beugung des asensiblen rechten Beines an. Inzwischen ist das linke Bein wieder in Beugestellung zurückgekehrt, und das rechte wird wieder gestreckt. Die Beine bleiben nun einige Zeit ruhig hängen, bis ein neuer Zyklus beginnt ${ }^{1}$. Von Anfang Mai ab sind alle möglichen Reflexe auf das asensible Bein mit grosser Leichtigkeit auszulösen: Schlag auf die linke Kniesehne, Kneifen der linken Zehenballen (gekreuzter Streckreflex), Spreizen der linken Zehen (Extensorstoss), passive Beugungen und Streckungen des linken Beines oder einzelner seiner Gelenke, Kneifen des Schwanzes und viele andere an den sensiblen Körperstellen angebrachten Reize bewirken deutliche und kräftige Reflexbewegungen des asensiblen Beines. Reiben der Bauchhaut rechts kranialwärts von der asensiblen Zone bewirkt deutlichen Kratzreflex des rechten Beines. Es ist also bei unverändert bestehender sensibler Lähmung des rechten Beines seine Motilität in vorzüglicher Weise zurückgekehrt und ist jedenfalls ebenso lebhaft wie bei gewöhnlichen Rückenmarkshunden mit intakten hinteren Wurzeln.

Nach der Durchtrennung des Rückenmarkes wurde der Hund im Laufe von 4 Monaten an 37 verschiedenen Tagen genau untersucht und an ihm alle die in der ersten Mitteilung ausführlich geschilderten Versuche über Schaltung und Reflexumkehr angestellt. Das Ergebnis war, dass in keinem einzigen unter vielen hundert Reflexversuchen sich irgendein Einfluss der Lage und Stellung, die dem rechten Beine gegeben wurde, a uf die Reflexbewegungen des rechten Beines nachweisen liess. Die Durchschneidung der hinteren Wurzeln hatte alle die beschriebenen Schaltungen vollkommen aufgehoben.

Im einzelnen ergaben sich folgende Verhältnisse, wobei immer auf die Schilderung der normalen Versuchsergebnisse in der ersten Mitteilung zurückverwiesen sei.

Der gekreuzte Kniesehnenreflex (Schlag auf die linke Kniesehne, Bewegung des rechten Beines) war vom 12. März ab deutlich. In der überwiegenden Mehrzahl der Fälle trat Streckung des rechten Beines auf, einerlei ob das Bein vorher gebeugt oder gestreckt war. War Knie und Fussgelenk gestreckt, Hüfte

1) Diese Beobachtungen bestätigen die neuerdings von Sherrington (Flexion-reflex of the limb, crossed extension reflex and reflex stepping and standing. Journ. of Physiol. vol. 40 p. 28. 1910) gemachten Angaben und stehen im Gegensatz zu denen von Philippson (Sur les phénoménes consécutives à la section de la moelle et à l'ablation des racines posterieures. Bull. soc. roy. sc. med. et nat. Bruxelles. 2. Dec. 1907). Auch bei meinem Hunde. hörte das Pendeln nach Festhalten des linken, nicht aber des rechten Oberschenkels auf. 
gebeugt, so erfolgte Streckung der Hüfte. War das Bein in allen Gelenken gestreckt, so liess sich vor allem eine weitere energische Streckung des Knies wahrnehmen. An fünf verschiedenen Tagen (also nur als seltene Ausnahmen) liess sich beim gekreuzten Kniesehnenreflex Beugung des rechten Beines beobachten. An einem Tage trat diese ausnahmslos in allen Versuchen ein, einerlei ob das rechte Bein sich in maximaler Beuge- oder Streckstellung befand. An vier anderen Tagen wurden sowohl Beuge- wie Streckbewegungen erhalten, regellos durcheinander und ohne dass die dem rechten Beine gegebene Stellung darauf irgendeinen Einfluss ausübt. So erfolgt z. B. sehr deutlich bei gebeugtem Beine weitere Beugung, mancbmal auch Adduktion dazwischen. Auch Beugung der rechten Hüfte zusammen mit Streckung des Knies wird beobachtet. Diese Tatsachen zeigen, dass bei dem Hunde sehr wohl die Möglichkeit besteht, beim gekreuzten Kniereflex mit Beugung oder Streckung zu reagieren, dass aber trotzdem die Schaltung durch veränderte Stellung des Gliedes nicht eintritt.

Auf Kneifen der linken Pfote trat ausnahmslos der gekreuzte Streckreflex ein. Ebenso erfolgte beim Auslösen des Extensorstosses links immer Streckung des rechten Beines, einerlei in welcher Ausgangsstellung sich das Bein befand.

Schnelle kräftige Beugung des tonisch gestreckten linken Knies bewirkte an zwölf verschiedenen Prüfungstagen Streckung des rechten Beines, wobei sich besonders das Kniegelenk beteiligte, an zwei Tagen wurde auch eine Adduktionsbewegung des Hüftgelenkes beobachtet, an einem Tage waren nur Beugereaktionen des rechten Beines zu erhalten, und an einem anderen Tage traten abwechselnd Beuge- und Streckreflexe auf, welche aber keine Abhängigkeit von der vorher dem Gliede gegebenen Stellung erkennen liessen.

Streckung des gebeugten linken Krnies hatte meist keine gekreuzten Reflexe zur Folge. An drei Tagen wurden Streckbewegungen, an einem Tage Beugebewegung rechts, vor allem des Knies erhalten, unabhängig von der Stellung des Gliedes.

Schnelle Streckung des gebeugten linken Beines hatte an einem Tage immer und bei jeder Stellung Streckung, an drei anderen Tagen immer Bengung des rechten Beines zur Folge, an drei Tagen trat Adduktion im Hüftgelenk ein, an einem weiteren Tage abwechselnd Beugung und Streckung, ohne dass ein Einfluss der Stellung des rechten Beines sich hätte nachweisen lassen. An zwei Tagen endlich erfolgte, einerlei welches auch die Ausgangsstellung war, immer zuerst eine Beugung, der dann eine Streckung folgte; d. $h$. das rechte Bein führte einen ganzen Schritt aus.

Reiben der Bauchhaut kopfwärts von der asensiblen Zone bewirkte stets und in zahlreichen Versuchen Beugung des rechten Beines, welches auch dessen Ausgangsstellung war. An diese Beugung schloss sich dann in vielen Fällen ein typischer Kratzreflex an.

Die angeführten Tatsachen zeigen, dass bei dem Hundedas rechte asensible Bein zu allen möglichen Reflexbewegungen beafähigt war. Trotzdem liess sich eine Schaltung durch veränderte Lage und Stellung des Gliedes nicht herbeiführen. Dieses ist um so bemerkenswerter, als beibestimmten Reflexenan einzelnen Tagen sehr wohl Beuge-wie Streckbewegungenzuerhalten waren; woraus sichergibt, dass beide Reaktionen sehr gut hätten ein- 
treten können. Trotzdem liess sich in keinem einzelnen Falle ein Einfluss der Stellung des Gliedes auf den Reizerfolg nachweisen.

Re sü mee: Bei einem Hunde wird nach einseitiger intraduraler Durchschneidung aller Hinterwurzeln vom 1. Lumbalis abwärts die rechte Pfote samt der angrenzenden Haut des Rumpfes asensibel. Die Motilität stellt sich nach einiger Zeit in befriedigender Weise wieder her. Nunmehr wird das Rückenmark am 11. Brustwirbel durchtrennt. Nachdem die Shokerscheinungen geschwunden sind, pendelt der Hund mit beiden Hinterbeinen, von den sensiblen Körperstellen sind alle möglichen Beuge- und Streckreflexe auf das asensible Bein zu erhalten, es lässt sich aber ein schaltender Einfluss der Lage und Stellung des Gliedes auf die Richtung der Reflexbewegung in keinem einzigen Falle mehr nachweisen.

\section{Versuch 2.}

„Eva", weibl. junger Foxterrier.

13. November 1909. In Ätherchloroformnarkose wird der Wirbelkanal vom Unterrand der letzten Rippe bis weit ins Kreuzbein hinein eröffnet, die Dura gespalten und rechterseits die Hinterwurzeln intradural vom ersten Lumbalnerven bis zum Schwanzmark durchtrennt. Operationsdauer $1 \frac{1 / 4}{4}$ Stunden. Nach dem Erwachen aus der Narkose ist der Patellarreflex links deutlich, rechts fehlt er.

22. November. Versuche zu laufen. Gekreuzte Reflexe von links nach rechts. Willkürbewegungen des rechten Hinterbeines.

13. Dezember. Erste kinematographische Aufnahme von Stehen und Laufen. Das Tier steht auf vier Pfoten, wobei oft längere Zeit auf dem Fussrücken des rechten Hinterbeines, ohne die Stellung zu korrigieren. Das asensible Bein wird oft abwechselnd gestreckt und gebeugt. Beim Gehen kann das asensible rechte Hinterbein zusammen mit dem linken Vorderbein kurze Zeit das Körpergewicht tragen. Beim Sitzen wird das asensible Bein meist tonisch gestreckt gehalten. Beim Laufen und Galoppieren beteiligt sich dasselbe und kann dabei wirksam mithelfen. Die Ataxie, das Ausfahren nach allen Richtungen ist aber so stark, dass der Hinterkörper gelegentlich zu Falle kommt und dann nachgeschleift wird.

29. Dezember. Zweite kinematographische Aufnahme. Beim Stehen wird der Stand auf dem asensiblen Fussrücken nicht korrigiert. Beim Traben und Galoppieren entwickelt der Hund eine ausserordentliche Lebhaftigkeit, er kommt dabei nur selten zu Falle. Auch beim Laufen tritt dabei die linke hintere Pfote oft mit dem Fussrücken auf, das ganze Bein macht noch stark ausfahrende Bewegungen und kommt dabei oft auf die linke Seite hinüber (Kreuzen). Beim Galoppieren wird die Pfote zum Abspringen kräftig mit benutzt. - Resü mee; Das asensible Bein hat nach der Operation eine vorzügliche Motilität wiedererlangt.

24. Januar 1910. Bestimmung und Photographie der Sensibilitätsgrenze (s. Fig. 2). Das Ergebnis stimmt mit den Bestimmungen vom 30. November, 
vom 10. Dezember und vom 24. Dezember 1909 vollkommen überein. Das ganze rechte Hinterbein ist asensibel, Patellar- und Beugereflex sowie Extensorstoss fehlen. Auf der ganzen Haut des Beines sind keine sensiblen Inseln zu entdecken. Auch die angrenzenden Partien des Rumpfes sind asensibel und zwar in etwas grösserer Ausdehnung als bei Hund "Gerda“ (Versuch 1). Die Grenze verläuft am Bauch in der Linea alba, an der Vulva, dem Damm, der medialen und dorsalen Seite des Schwanzes und am Rücken genau in der Mediallinie. Die kraniale Grenzlinie verläuft ungefähr in einer Frontalebene, und zwar beginnt sie zwei Finger breit unter dem Nabel, geht an den oberen Ansatz der Schenkelfalte und von da etwa drei Finger breit unterhalb des Rippenbogens und zwei Finger breit oberhalb des Darmbeinkammes gegen die Wirbelsäule. - Diese Sensibilitätsgrenze hat sicb während der ganzen ferneren Dauer des Versuches nicht geändert.
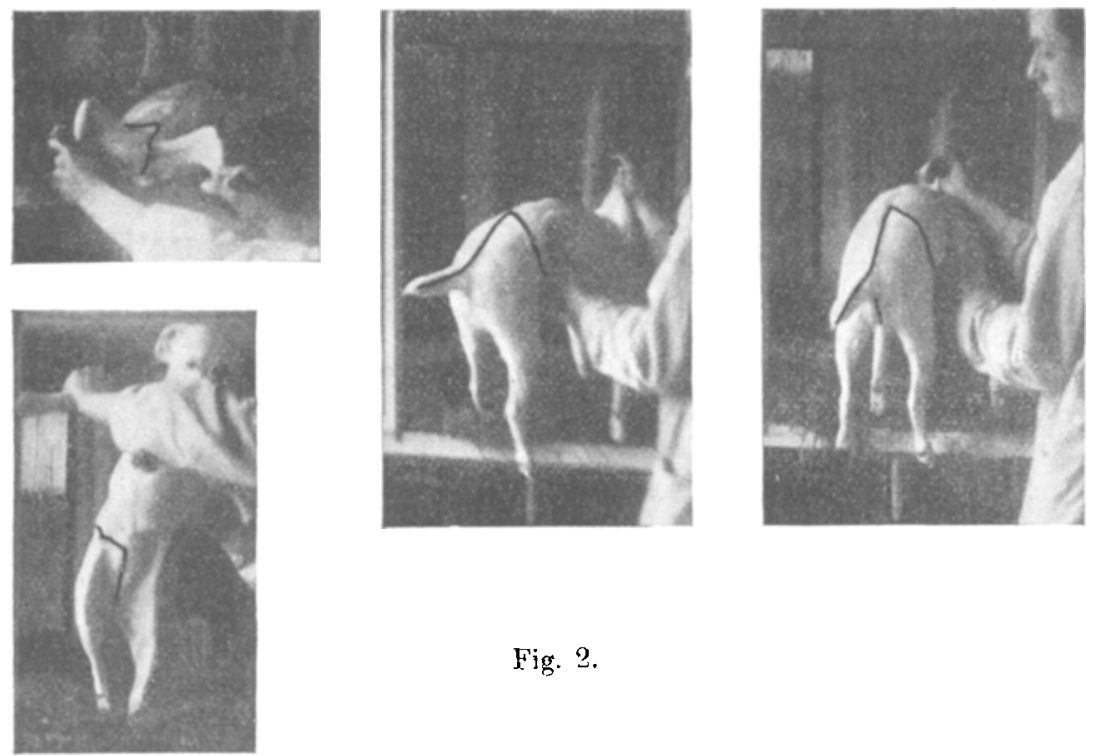

Fig. 2.

Auch hier wieder verdanke ich Herrn Prof. Winkler die Aufklärung, wie die gefundene Sensibilitätsgrenze zu der beabsichtigten Operation stimmt. Der Hund hat nach dem Ergebnis der Röntgenuntersuchung dreizehn rippentragende Brustwirbel und sechs Lendenwirbel, die Hinterextremität ist gegenüber dem Rückenmark in "präfixer" Stellung. Aus diesen Befunde ergibt sich im Zusammenhang mit den Befunden Winkler's und v. Rynberk's, dass ausser sämtlichen caudalen, sakralen und lumbalen auch die 13, thorakale Hinterwurzel höchstwahrscheinlich noch mit durchtrennt ist. Nach dem, was bei Gelegenheit der Besprechung von Versuch 1 angeführt wurde, ist es danach sicher, dass auch alle mit der hinteren Extremität in Beziehung stehendén Muskeln ihrer afferenten Bahnen völlig beraubt sind.

Aus dem Vorhergebenden folgt; dass durch dievorgenommene Hinterwurzeldurchschneidung das ganze rechte Hinterbein mit 
allen Teilen, welche bei seinen Bewegungen in Mitleidenschaft gezogen werden können (Haut, Muskeln etc.), asensibel gemacht worden ist.

Da ausserdem die Extremität eine ganz vorzügliche aktive Motilität in allen Gelenken und nachallen Richtungenhinzeigte, so schien nunmehr die Zeit für die Querdurchtrennung des Rückenmarkes gekommen, welche am 26. Januar 1910 in Ätherchloroformnarkose am 10. oder 11. Brustwirbel vorgenommen wurde. Fine Stunde nach der Operation war links ein starker Patellarreflex nachzuweisen. Auf Kneifen der linken Hinterpfote trat Bewegung der Zehen des rechten asensiblen Beines auf.

27. Januar. Schwacher Extensorstoss rechts. Dasselbe am 1. Februar, wo auch Spontanbewegungen beider Beine und deutliche gekreuzte Reflexe von links nach rechts wahrgenommen wurden. Von nun an stellen sich allmählich die Reflexe wieder her, und die Shokerscheinungen schwinden. Bis Anfang Juli 1910 werden an 34 verschiedenen Tagen im ganzen 93 protokollierte Finzelversuche vorgenommen, bei denen sich die verschiedenen Reflexe auf das rechte (asensible) Bein im einzelnen in folgender Weise verhielten:

Bei Schlag auf die linke Kniesehne erfolgte anfangs meist Beugung, von Mitte März ab dagegen ausnahmslos Streckung, am stärksten im Knie, meist auch in den anderen Gelenken, manchmal begleitet von einer Adduktion im Hüftgelenk. - Kneifen der linken Pfote bewirkte Streckung, am stärksten im Kniegelenk, meist wurden auch die anderen Gelenke gestreckt, nur an einem Tage wurde Beugung des Fussgelenkes, an einem anderen des Hüftgelenkes notiert. Wurde am linken Beine der Extensorstoss ausgelöst, so erfolgte anfangs Streckung des rechten Beines. Von Mitte April ab wurde konstant eine sehr merkwürdige komplizierte Bewegung beobachtet, die aus Adduktion der Hüfte, Streckung des Knies und anfänglicher Beugung des Fusses mit nachfolgender Streckung desselben zusammengesetzt war und zu einer Art Herumschleudern des Beines gegen den Bauch zu Anlass gab. Diese Bewegung trat ein, einerlei welches auch. die Ausgangsstellung des rechten Beines war. - Beugung des tonisch gestreckten linken Knies bewirkte fast immer eine Adduktion der rechten Hüfte, wozu sich manchmal eine Streckung von Hüfte und Krnie gesellte. - Beugung des gestreckten linken Beines in allen Gelenken führte zu Streckung des rechten Knies. Streckung des gebeugten Knies bewirkte meist Beugung rechts. An einem Tage trat regellos Beugung oder Streckung ein, ohne dass sich hierauf ein Einfluss. der Stellung des rechten Beines hätte nachweisen lassen. - Streckung der gebeugten linken Hüfte wurde von Beugung der rechten Hüfte gefolgt. - Kneifen des Schwanzes bewirkte eine Beugung beider Beine in allen Gelenken, an welche sich nach dem Aufhören des Reizes eine kräftige Streckbewegung in allen Gelenken anschloss. - Reiben der Bauchhaut linkerseits führte zu Beugung des. rechten Fusses.

Mit beiden Beinen werden beim Hängen Spontanbewegungen ausgeführt, dieselben steigern sich bei längerdauerndem Kneifen des Schwanzes zu lebhaften Zappel- und Strampelbewegungen, welche aber nicht so regelmässig alternieren, dass man von richtigem „Pendeln" sprechen könnte. Der Tonus des asensiblen. 
Beines ist meist geringer als der des anderen. An einzelnen Tagen konnte aber ein deutlicher Unterschied nicht festgestellt werden.

Die angeführten Tatsachen zeigen, dass das asensible Bein des Rückenmarkshundes eine vorzügliche Motilität besitzt, dass Reflexbewegungen in allen Gelenken und in allen Richtungen an ihm hervorgerufen werden können. Trotzdem war das Ergebnis in bezug auf die uns hier interessierende Frage ein eindentig negatives. Niemals trotz vielfacher Bemühungen habe ich auch nur eine Andeutung davon aufinden können, dass die Lage und Stellung, die dem rechten Beine vor Auslösung eines Reflexes gegeben wurde, von irgendeinem Einfluss auf die Richtung war, in welcher diese Reflexbewegung erfolgte. Das Resultat war also vollkommen das gleiche wie bei dem Hunde von Versuch I. Die Durchschneidung der hinteren Wurzeln hatte alle die in der ersten Mitteilung beschriebenen Schaltungen völlig aufgehoben.

Res ümee: Bei einem Hunde wird nach einseitiger intraduraler Durchschneidung aller lumbalen und sakralen Hinterwurzeln die rechte Pfote samt den angrenzenden Teilen der Rumpfhaut asensibel. Die Motilität stellt sich nach einiger Zeit in befriedigender Weise wieder her. Nunmehr wird das Rückenmark am 10.-11. Brustwirbel durchtrennt. Nachdem die Shokerscheinungen geschwunden sind, können alle möglichen Beuge- und Streckreflexe auf das asensibele Bein hervorgerufen werden; es lässt sich aber ein schaltender Einfluss der Lage und Stellung des Gliedes auf die Richtung der Reflexbewegung in keinem einzigen Falle mehr nachweisen.

\section{b) Versuche an Katzen.}

Die Versuche an Katzen bezwecken, festzustellen, ob die in der zweiten Mitteilung ${ }^{1}$ ) beschriebenen Schaltungsreaktionen nach Durchschneidung der hinteren Wurzeln noch nachzuweisen sind oder nicht. Es handelte sich dabei um Katzen, die zuerst in tiefer Chloroformnarkose dezerebriert waren, und denen $1 / 2-1$ Stunde danach das Rückenmark in der Höhe des 10.-12. Brustwirbels durchtrennt wurde. Dann entwickelte sich in kurzer Zeit eine hochgradige Reflexerregbarkeit des hinteren Rückenmarksabschnittes, wobei es auch zu sehr leicht auslösbaren und sehr kräftigen Reflexbewegungen des Schwanzes kam. Auf leichtes Kneifen der Schwanzspitze oder auch nur auf Berührung der Haare an dieser Stelle wird der Schwanz, wenn er sich in symmetrischer Lage zur Körperachse be-

1) R. Magnus, Zur Regelung der Bewegungen durch das Zentralnervensystem. II. Mitt. Pflüger's Arch. Bd. 130 S. 253. 1909. 
findet, regellos nach allen Seiten bewegt. Sowie aber der Schwanz nach irgendeiner Seite von der Mittellinie abgelenkt wird, so verschwindet die Regellosigkeit, und der Schwanz schlägt immer nach der gedehnten Seite. Es wurden eine ganze Reihe von verschiedenen Versuchsanordnungen geschildert, welche alle diese Regel veranschaulichen. In der anatomischen Anordnung liegt es begründet, dass die seitliche Ablenkung des Schwanzes die auschaulichsten Resultate gibt. Die Bewegungen erfolgen, wenn der Schwanz als Ganzes in einer Richtung von der Mittellinie abgelenkt wird, vor allem an der Schwanzwurzel. Daher seien einige Bemerkungen über die anatomische Anordnung der Abduktoren (Seitwärtswender) des Schwanzes bei der Katze hier angeführt, wohei für ein genaueres Studium auf das Werk von Strauss-Durkheim ${ }^{1}$ ) und auf die vergleichende Untersuchung von $\mathrm{Eggeling}^{2}$ ) verwiesen sei.

1. Der wichtigste Abduktor der Schwanzwurzel ist M. spino-caudalis (Eggeling) - Ischio-caudal (Strauss-Durkheim) - M. abduktor caudae internus s. coccygeus (Ellenberger und Baum). Derselbe verläuft von der Spina ischiadica zu den Querfortsätzen der vier ersten Schwanzwirbel.

2. Ein weiterer Abduktor der Schwanzwurzel ist der Abduktor cocygis ext. (Ellenberger und Baum) - Longs-sus-intertransversaires de la quene (Strauss-Durkheim). Dieser entspringt am Becken an der Innenseite des Os Ilium gegenüber den Sakralwirbeln, ferner an den Seiteuteilen der Sakralwirbel selber und an den Seitenteilen der ersten Schwanzwirbel. Der Muskel inseriert an den Querfortsätzen der zwei letzten Sakral- und der vier ersten Schwanzwirbel. Entsprechende Muskeln setzen sich noch bis zum 9. Schwanzwirbel, allmählich schwächer werdend, fort.

Ausser diesen hauptsächlich die Schwanzwurzel abduzierenden Muskeln sind besonders von Strauss-Durkheim noch eine ganze Reihe von Muskeln beschrieben worden, welche mehr für die Abduktion des Schwanzes in seinem weiteren Verlaufe bestimmt sind, und welche in der Nomenklatur dieses Autors hier aufgezählt seien.

3. Longs-sous-plagio-transversaires und Longs-plagio-mamillaires (entsprechen zum Teil dem M. sacro caudalis von Eggeling) entspringen an der Innenseite des Beckens von den drei sakralen Querfortsätzen und ferner von den Seitenteilen der zehn ersten Schwanzwirbel. Sie inserieren sich an den Seitenteilen der Schwanzwirbel vom 5. abwärts bis zur Schwanzspitze.

1) H. Strauss-Durkheim, Anatomie descript. et compar. du chat. Paris 1845 .

2) H. Eggeling, Zur Morphologie der Dammmuskulatur. Morph. Jahrb. Bd. 24 S. 405.1896. 
Zur Regelung der Bewegungen durch das Zentralnervensystem. III. $\quad 559$

4. Moyen-sous-plagio-transversaires entspringen lateral vom 2. und den folgenden Schwanzwirbeln und inserieren vom 6. Schwanzwirbel an abwärts.

5. Courts-sous-plagio-transversaires und Interplagiens caudaux sind kurze Muskeln zwischen den Seitenteilen der einzelnen Schwanzwirbel.

Die Schwanzwurzel selber liegt bei der Katze dem Blicke verborgen am Ende des Rückens. Erst der 5. Schwanzwirbel befindet sich vertikal über dem After, und erst der 6 . Wirbel liegt in demjenigen Teil, der dem unbefangenen Beobachter des Tieres als der Beginn des Schwanzes erscheint.

Die Kenntnis der segmentalen motorischen Innervation des Katzenschwanzes verdanken wir hauptsächlich Sherrington ${ }^{\mathbf{l}}$ ), der an 27 Katzen die vorderen Wurzeln gereizt hat.

Er fand bei Tieren mit postfixem Typus des Plexus Iumbosacralis auf Reizung der

8. postthorakalen (1. sakr.) Wurzel: 9

10.

(2., ) ,

Abduktion der Schwanzwurzel nach der (3. ") ") gereizten Seite.

Bei Tieren mit präfixer Anordnung des Plexus ergab sich auf Reizung der

7. postthorakalen (7. humb.) Wurzeln Bewegung der Schwanzwurzel nach der Reizseite.

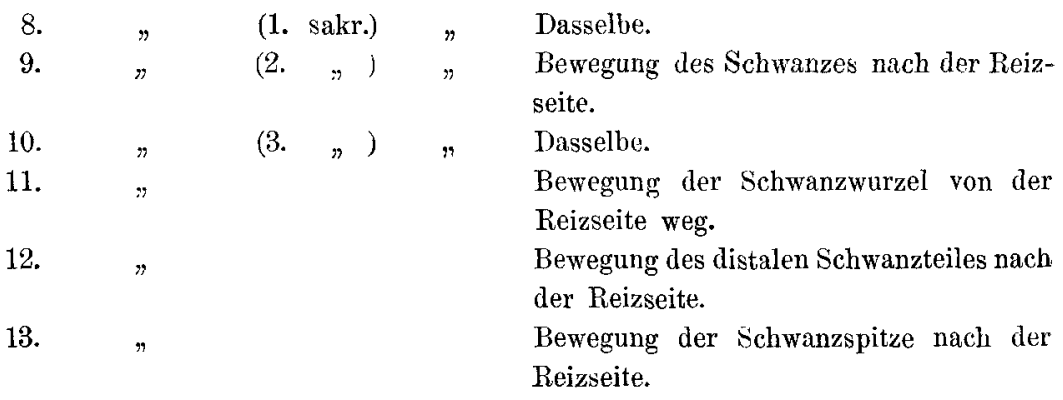

Wie man sieht, kommen für die Bewegung der Schwanzwurzel das Sakralmark und die unmittelbar angrenzenden lumbalen und coccygealen Abschnitte des Rückenmarkes in Betracht. In diesem Gebiete muss also die Hinterwurzeldurehsehneidung für den hier vorliegenden $\mathrm{Z}$ weck vorgenommen werden.

Wie oben erwähnt wurde, diente als wichtigster Ort für die Auslösung der Schwanzreflexe die äusserste Schwanzspitze. Diese durfte also durch die Hinterwurzeldurchschneidung nicht mit aseusibel

1) C. S. Sherrington, Notes on the arrangement of some motor fibres in the lumbo-sacral plexus. Journ. of Physiol. vol. 13 p. 621. 1892. 
gemacht werden. Es waren also die allerletzten sensiblen Wurzeln im Schwanzteile des Rückenmarkes bei der Operation zu schonen.

Danach ergab sich nun der folgende Versuchsplan. Bei Katzen wurde der Wirbelkanal vom 4 . oder 5. Lendenwirbel an abwärts unter Ätherchloroformnarkose eröffnet, mit Dura gespalten und die Hinterwurzeln beiderseits vom 5. Lumbalsegment abwärts durchtrennt, wobei nach Möglichkeit die allerletzten Wurzelfäden geschont wurden. Nach Heilung der Wunde wurde der Sensibilitätsausfall festgestellt und solange gewartet, bis der Schwanz und besonders die Wurzel wieder aktiv in kräftiger Weise nach allen Seiten bewegt werden konnte. Erst danach wurde dann die betreffende Katze dezerebriert und an ibr die Schwanzreflexe untersucht.

Bei allen operierten Tieren wurde After, Damm, Vulva bzw. Scrotum nebst den unmittelbar angrenzenden Partien von Bauch und Rücken asensibel gefunden. Patellar- und Pfotenreflexe verhielten sich verschieden. Der proximale Teil des Schwanzes war in verschiedener Ausdehnung unempfindlich. Diese Unterschiede dürften, was die kraniale Grenze anbetrifft, weniger auf Verschiedenheiten in der Ausführung der Operation als auf den hochgradigen Variationen beruhen, welche mit prä- und postfixem Typus des Lumbosakralplexus und mit Variationen der Wirbelsäule zusammenhängen.

Im ganzen verfüge ich über drei Katzen, bei welchen die Operation in befriedigender Weise gelungen ist. Ausserdem werde ich noch zwei Versuche referieren, von denen der eine einen vollständig asensiblen, der andere einen zu wenig asensiblen Schwanz ergab. Das Ergebnis aller dieser Fxperimente an Katzen ist dasselbe wie das der Hundeversuche. Nach Durchschneidung der hinteren Wurzeln sind die früher beschriebenen Schaltungen vollständig aufgehoben. Durch die Ablenkung des Schwanzes von der Medianlinie wird die Richtung der Reflexbewegung nunmehr in keiner Weise mehr beeinflusst und bestimmt.

Katze "Kees".

\section{Versuch 3.}

19. Dezember 1909. Ätherchlorofomnarkose. Eröffnung des Wirbelkanals vom 6. Lendenwirbel inkl. an abwärts. Intradurale Durchschneidung der beiderseitigen Hinterwurzeln bis ins Sakrum. Muskel- und Hautnaht. Borsäure-Kollodium-Verband. Heilung per primam. 
Zur Regelung der Bewegungen durch das Zentralnervensystem. III. 561

27. Januar 1910. Schwanzspitze $10 \mathrm{~cm}$ lang sensibel. Schwanzmitte und -wurzel $(14,5 \mathrm{~cm}$ lang) vollständig asensibel. Ebenso Damm, After, Vulva. Die Sensibilitätsgrenze ist an der Linea alba $20 \mathrm{~mm}$ kranialwärts von der Spitze der Vulva, an der Streckseite des Oberschenkels links $35 \mathrm{~mm}$, rechts $24 \mathrm{~mm}$ von der Spitze der Vulva. Sie zieht beiderseits lateral vom Tuber ischii nach dem Rücken hinüber und ist in der Gegend des Trochanter links $38 \mathrm{~mm}$, rechts $40 \mathrm{~mm}$ von der Schwanzwurzel entfernt (Fig. 3). Patellar- und Berührungsreflexe an den Pfoten beiderseits vorhanden. Schwanz wird beim Gehen mit normalem Tonus getragen, wird spontan nach allen Seiten lebhaft bewegt.

1. Februar 1910. Versuch. 9 Uhr 50 Min Dezerebrierung in Chloroformnarkose. Danach gute Enthirnungsstarre aller vier Beine. 10 Uhr 45 Min. Durchschneidung des Rückenmarks am 11. Brustwirbel. Sofort starke Reflexe des Hintertieres. In den folgenden drei Stunden werden nun sämtliche in der 2. Mitteilung unter Nr. 1-6 geschilderten Schaltungsversuche ${ }^{1}$ ) wiederholt angestellt.

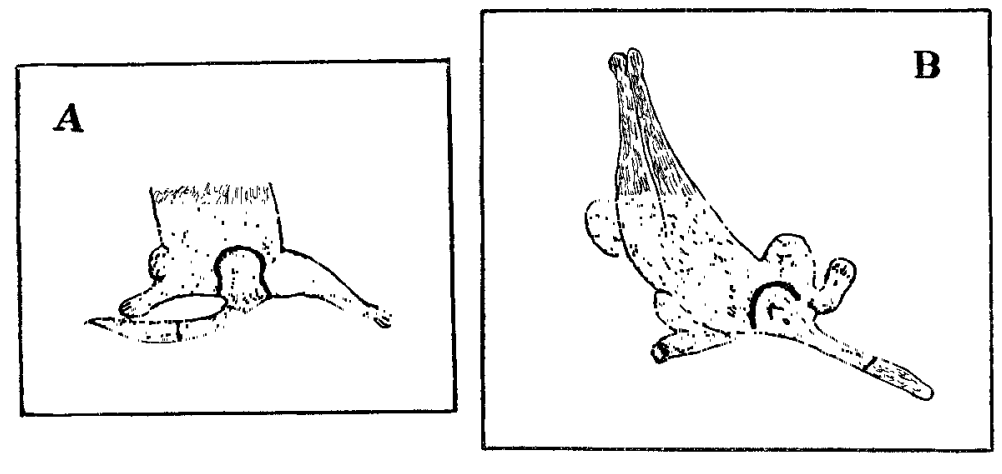

A Rückenansicht. Fig. 3. B Bauchansicht.

Das Ergebnis ist, dass der Schwanz auf Berührung seiner Spitze meist nach rechts schlägt, dazwischen aber auch gelegentlich ganz regellos nach links, dorsaloder ventralwärts. Ein Einfluss der Lage und der Krümmung des Schwanzes auf die Bewegungsrichtung ist dabei nicht nachzuweisen. Mit Hilfe der Reflexe wird darauf die Sensibilitätsgrenze nochmals bestimmt, mit weisser Farbe auf das Fell gezeichnet und nach dem Tode photographiert (Fig. 3).

Sektion: Wunde reizlos. Rückenmark nirgends verwachsen. Formolfixierung.

1) Nr. 1. Seitenlage. Schwanz hängt über den Tischrand: schlägt nach oben. - Nr. 2. Seitenlage. Schwanz über die Horizontale gehoben: schlägt nach unten. - Nr. 3. Seitenlage. Schwanz in der Mitte mit dem Finger gehoben: Wurzel schlägt nach unten oder Mitte nach oben. - Nr. 4. Seitenlage. Schwanz auf dem Tisch: schlägt nach oben. - Nr. 5. Hinterkörper symmetrisch, Bauch unten. Vorderkörper gedreht: Schwanz schlägt nach der Seite der Vorder beine. - Nr.6. Rückenlage. Schwanz anf dem Tisch, wird nach der Seite abgelenkt: schlägt nach der anderen Seite.

Pflüger's Archiv für Physiologie. Bd. 134. 
Katze "Mieze".

\section{Versuch 4.}

5. Februar 1910. Operation wie Versuch 3 vom 4. Lendenwirbel bis ins Sacrum. Gute Heilung.

5. März 1910. Schwanz tonisch nach oben getragen, kann aktiv nach beiden Seiten bewegt werden. Schwanzspitze bis etwas über die Mitte gut sensibel, proximale Hälfte völlig asensibel, ebenso die angrenzenden Teile der Bauch-, Rücken- und Oberschenkelhaut (s. Fig. 4). Patellarreflex rechts vorhanden, links abwesend. Beide Pfoten asensibel, das Tier hat sich am 17. Februar die linke Pfote bis zur Mitte des Unterschenkels abgefressen, was unter Perubalsam und einen Mull-Kollodiumstiefel zur Heilung gebracht wird. Läuft mit der anderen Pfote auf dem Fussrücken. Harnentleerung erfolgt wieder spontan.

10 Uhr 30 Min. Dezerebrierung in Chloroformnarkose. Nach Entwicklung guter Enthirnungsstarre Durchtrennung des Rückenmarks am 11. Brustwirbel.

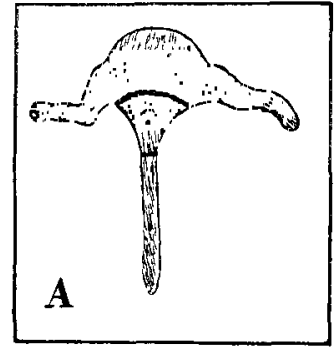

A Bauchansicht.

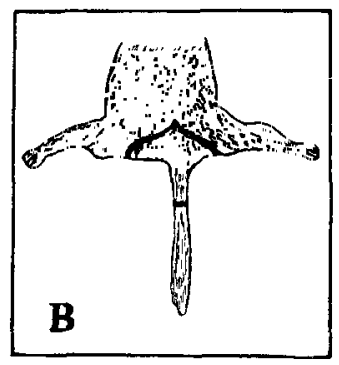

Fig. 4. B Rückenansicht.

In den folgenden $31 / 2$ Stunden werden die Schwanzreflexe fortlaufend geprüft (Versuche 1, 4, 5 und 6 der 2. Mitteilung). Das Ergebnis ist, dass die dem Schwanz gegebene Lage ohne jeden Einfluss auf die Richtung der Reflexbewegung ist. Dagegen ist ein deutlicher Einfluss der Reizstelle zu konstatieren, indem auf Kneifen der äussersten Schwanzspitze der Schwanz immer nach rechts, auf Berühren der Haare an der Dorsalseite des Schwanzes dagegen meist nach links schlägt. Gelegentliche Ventralflexionen werden auch beobachtet. Niemals aber hat die dem Schwanz gegebene Stellung und Krümmung irgendeinen Einfluss auf die Richtung des folgenden Reflexes. Die Sensibilitätsgrenze wird nochmals bestimmt, mit Farbe dem Tier auf das Fell gemalt und photographiert (Fig. 4).

Sektion: Wunde gut geheilt. Rückenmark nirgends verwachsen und in gutem Zustand. Formolfixierung.

Katze "Het " $\mathrm{Y}$ ".

\section{Versuch 5.}

13. April 1910. Operation wie Versuch 3, vom 5. Lendenwirbel inkl. bis Sacrum. Dabei leichte Verletzung der Hinterstränge in der Höhe des 5. Lendenwirbels (also ausserhalb der für die Schwanzbewegungen in Betracht kommenden Rückenmarksregion). Vorzügliche Heilung.

11. Mai 1910. Schwanz wird mit gutem Tonus getragen, wird sowohl an der Wurzel, wie an der Mitte und Spitze aktiv nach links, rechts, ventral- und dorsalwärts kräftig bewegt. Die Schwanzspitze ist sensibel, die Schwanzwurzel 
völlig asensibel, die Sensibilitätsgrenze liegt in der distalen Hälfte des Schwanzes. Vulva, Damm, Anus sowie die angrenzenden Partien von Bauch, Rücken und Oberschenkel asensibel (Fig. 5). Patellarreflex rechts vorhanden, links abwesend. Beide Pfoten asensibel. Das Tier läuft stark ataktisch und meist auf den beiden Fussrücken. Blase wird nur selten spontan entleert.

11 Uhr 40 Min. Dezerebrierung in Chloroformnarkose. Nach Entwicklung der Enthirnungsstarre wird 12 Uhr 12 Min. das Rückenmark am 13. Brustwirbel durchtrennt. Darauf sofort lebhafte Reflexe bei Berühren der Schwanzspitze. In den folgenden $2^{1 / 2}$ Stunden werden die Schwanzreflexe (Versuch 1, 2, 3, 4, 5 und 6 der 2. Mitteilung) fortlaufend geprüft. Dabei erfolgt auf Berühren der Schwanzspitze sowohl wie auf Reizung von anderen Körperstellen (z. B. Druck

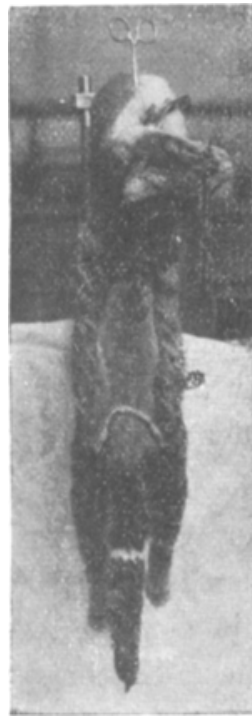

Fig 5 A. Rückenansicht. (Auf $1 / 2$ verkleinert.)

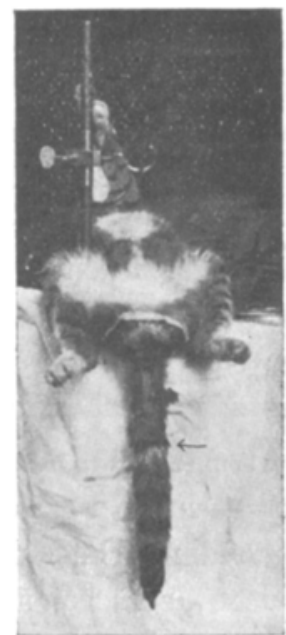

Fig. 5 B. Bauchansicht. (Auf $1 / 2$ verkleinert.)

auf die Lendenwirbelsäule) kräftiges Schlagen nach rechts, links, dorsal- oder ventralwärts. Manchmal wird die Schwanzmitte auch nach einer anderen Richtung bewegt als die Wurzel. In keinem Falle ist irgendein Einfluss der dem Schwanze vorher gegebenen Lage und Krümmung auf die Richtung der nachfolgenden Reflexbewegung nachzuweisen. Der nach links abgebogene Schwanz z. B. schlägt ebenso oft und kräftig nach links wie nach rechts. - Markierung der Sensibilitätsgrenze mit Farbe auf dem Fell des Tieres (Photographie s. Fig. 5).

Sektion: Wunde tadellos geheilt. Im Rückenmark befindet sich in der Höhe des 5. Lendenwirbels ein kleiner dorsaler Erweichungsherd, dort auch die Dura etwas verwachsen. Ubriges Rückenmark in gutem Zustande. Formolfixierung.

Resümee von Versuch 3-5: Bei drei Katzen wurden intradural beiderseits die Hinterwurzeln im Sakralmark und den 
angrenzenden lumbalen und coccygealen Rückenmarksabschnitten durchtrennt, die Wunde wurde zur Heilung gebracht, der Sensibilitätsausfall genau bestimmt. Derselbe betraf Damm, Vulva, Anus und die angrenzenden Partien von Bauch, Rücken und Oberschenkeln sowie die proximale Hälfte des Schwanzes. Bei zwei Tieren waren auch beide Pfoten asensibel. Die Motilität des Schwanzes stellte sich gut wieder her, derselbe wurde mit deutlichem Tonus getragen und wurde aktiv nach allen Seiten mit Kraft bewegt. 4-6 Wochen nach der Operation wurde mit diesen Tieren dann der in der zweiten Mitteilung beschriebene Versuch zur Untersuchung der Schaltungen bei den Schwanzreflexen angestellt, die Tiere wurden in Narkose dezerebriert, nach Entwicklung der Enthirnungsstarre das Rückenmark im unteren Dorsalteile durchtrennt und nunmehr bei verschiedenen Lagen des Tieres und des Schwanzes nachgesehen, ob die Lage, Stellung oder Krümmung des Gliedes irgendeinen Einfluss auf die Richtung der Reflexbewegungen besitzt. Das Ergebnis war in allen Versuchen ein eindeutig negatives. Der Schwanz wurde wohl bei den Reflexen kräftig nach allen Seiten bewegt, aber ganz regellos, und es war nicht mehr möglich, wie das in den Normalversuchen stets der Fall ist, die Richtung der eintretenden Reflexbewegung mit Sicherheit vorauszusagen.

Im Anschluss an diese drei vollkommen gelungenen Versuche sei dann noch über zwei Experimente berichtet, bei denen die Hinterwurzeldurchschneidung nicht genau die gewollte Ausdehnung hatte, welche aber geeignet sind, das allgemeine Ergebnis weiter zu befestigen.

Katze "Zebra".

\section{Versuch 6.}

12. April 1910. Operation wie Versuch 3, vom 5. Lendenwirbel bis ins Sacrum. Glatte Heilung.

6. Juni 1910. Schwanz wird mit gutem Tonus getragen, wird an der Wurzel und an seinem ganzen Verlauf nach allen Richtungen hin prompt und kräftig bewegt. Asensibel sind Anus, Damm, Vulva sowie die angrenzenden Partien von Bauch und Rücken. Der ganze Schwanz ist asensibel, so dass sich das Tier am 21. Mai die äusserste Schwanzspitze anfrisst. Die Wunde wird mit dem Termokauter behandelt und ist am 28. Mai geheilt. Patellarreflexe und Berührungsreflexe an beiden Pfoten deutlich vorhanden. Blase wieder spontan entleert.

$10 \mathrm{Uhr} 13 \mathrm{Min}$. Dezerebrierung in Chloroformnarkose. Nach Entwickelung der Enthirnungsstarre wird 11 Uhr 20 Min. das Rückenmark am 12. Brustwirbel durchtrennt. In den folgenden drei Stunden werden die Schwanzreflexe 
Zur Regelung der Bewegungen durch das Zentralnervensystem. III. 565

fortlaufend geprüft (Versuch 1, 2, 4, 5 und 6 der 2. Mitteilung). Da aber der ganze Schwanz asensibel ist, müssen die Reize an anderen Körperstellen angebracht werden, vorzugsweise am Rücken und den Pfoten. Dabei erfolgt auf Drücken der Lendenwirbelsäule immer Schlagen des Schwanzes nach links, manchmal nit gleichzeitiger Depression der Schwanzwurzel. Auf Berühren der Haut am Rücken seitlich neben der Wirbelsäule schlägt der Schwanz immer nach der gereizten Seite, also bei Berührung der rechten Flanke nach rechts, der linken Flanke nach links. An diesem Ergebnis ändert es nichts, wenn man den Schwanz auch noch so sehr nach einer Seite von der Mittellinie abbiegt, oder wenn er bei Seitenlage des Tieres über den Tischrand nach unten herabhängt. Die Richtung der Reflexbewegung ist völlig unabhängig von der Lage und Stellung des Schwanzes und wird allein durch Art und Ort des Reizes bedingt. Aufzeichnung und Photographie der Sensibilitätsgrenze.

Sektion: Wunde glatt geheilt. Dura bis zum Unterrande des 4. Lendenwirbels eröffnet. Dort etwas Verwachsung, ebenso wie an einer kleinen Stelle im Sacrum. Im Übrigen Rückenmark frei und in gutem Zustande. Formolfixierung.

Resümee: Der Versuch stimmt im allgemeinen mit den früheren Experimenten überein, nur war der Schwanz in seiner ganzen Länge asensibel, und es konnten daher die Reize nicht an der Schwanzspitze angebracht werden. Auch in diesem Falle liess sich irgendein Einfluss der Lage und Stellung des Gliedes auf die Richtung der Reflexbewegung nicht nachweisen.

Kater "Velox".

Versuch 7.

12. April 1910. Operation wie Versuch 3, vom 5. Lendenwirbel bis ins Sacrum. Danach Abszess am Rücken, der am 4. Mai geheilt ist.

28. Juni 1910. Schwanz wird mit Tonus getragen, kann aktiv nach allen Seiten bewegt werden. Der ganze Schwanz ist sensibel mit Ausnahme von einer Strecke von $2 \mathrm{~cm}$ an der Schwanzwurzel. Damm, Anus, Penis asensibel, ebenso die Hinterseite des Oberschenkels und die Pfoten. Am Bauche liegt die Sensibilitätsgrenze $1 \mathrm{~cm}$ vor dem Penis, am Rücken $1 \mathrm{~cm}$ vor einer die Trochanteren verbindenden Linie. Patellarreflexe abwesend. Starke Ataxie der Hinterbeine beim Laufen. Das Tier läuft auf dem Rücken der Pfoten und Unterschenkel, kreuzt dabei oft medialwärts mit den Beinen nach der anderen Seite hinüber, kann sich aber auf den Hinterbeinen erheben und sogar aus dem Käfig springen. Harnentleerung erfolgt wieder spontan.

10 Uhr 33 Min. Dezerebrierung in Chloroformnarkose. Nach Entwicklung der Enthirnungsstarre um $11 \mathrm{Uhr}$, Durchschneidung des Rückenmarkes am 11. Brustwirbel. Daranf sofort gute Schwanzreflexe auf Berührung der Schwanzspitze. In den folgenden 4 Stunden werden dieselben fortlaufend geprüft. Die Bewegungen des Schwanzes erfolgen dabei nach allen Seiten kräftig, und zwar treten Bewegungen an der Wurzel, der Mitte und der Spitze auf. Oft wird die Mitte nach einer anderen Richtung bewegt, als die Wurzel. Bei einer ganzen 
Reihe von Versuchen ist nun mit Sicherheit die Abwesenheit jeder Art von Schaltung zu erkennen. Und zwar gilt das für alle diejenigen Versuche, bei denen der Schwanz als Ganzes eine gerade Linie bildet und nur an seiner Wurzel von der Richtung der Körperachse abgebogen ist (Versuch 2, 4, 5 und 6 der II. Mitteilung). Wenn z. B. das Tier auf den Rücken gelegt wird, der Schwanz ebenfalls auf dem Tisch liegt und an der Wurzel nach rechts oder links abgebogen wird, so ist dadurch nicht wie in den Normalversuchen die Richtung der Reflexbewegung eindeutig bestimmt, sondern es erfolgen ganz regellose Bewegungen nach allen Seiten. Wenn aber das Tier auf die Seite gelegt wird und der Schwanz über den Tischrand nach unten hängt (vgl. Fig. 1 und 2 der II. Mitteilung), so ist nicht nur die Schwanzwurzel gegen die Körperachse abgebogen, sondern das proximale Drittel des Schwanzes selber ist gekrümmt. Diese Kŕrümmung fällt nun bei der Katze Velox in den sensibelen Bezirk, und damit hängt es zusammen, dass bei diesem und $n$ ur bei diesem Versuche sich nun ein deutlicher, wenn auch nicht sehr hochgradiger Einfluss der Stellung des Schwanzes auf die Richtung der Reflexbewegung erkenren lässt. Bei linker Seitenlage erfolgt auf Berühren der Spitze meist Schlag nach rechts (oben), be $\mathrm{e}^{\mathrm{i}}$ rechter Seitenlage nach links (oben). Die Schaltung ist aber keine absolut sichere wie in den Normalversuchen, sondern gelegentlich kommen auch Fehlreaktionen vor.

Sektion: Abszess völlig geschwunden. Dura bis zum Oberrande des 5. Lendenwirbels eröffnet. Dort geringe Verwachsung des Rückenmarkes mit der Umgebung, welches im übrigen aber frei und reizlos gefunden wird. Formolfixierung ${ }^{1}$ ).

Resùmee: Bei dieser Katze ist der Ausfall der Sensibilität etwas weiter kranialwärts erfolgt, als beabsichtigt war. Der Schwanz blieb in seinem grössten Teile sensibel, und nur ein kleines Stück der Schwanzwurzel war zusammen mit dem Hinterende des Rumpfes und einem Teile der Hinterbeine gefühllos geworden. Das Ergebnis war nun ein recht interessantes. Alle Lagen, in denen der Schwanz als ganzes gerade und ungekrümmt gelassen und nur seine Wurzel gegen die Körperachse abgebogen wurde, waren ohne jeden schaltenden Einfluss, und die Reflexbewegungen erfolgten ganz regellos in allen Richtungen. Wurde dagegen nicht nur die Schwanzwurzel allein, sondern auch das proximale Drittel des Schwanzes mit abgebogen, so war nunmehr ein schaltender Einfluss deutlich, so dass der Schwanz nun wenigstens in der Mehrzahl der Fälle nach der gedehnten Seite schlug. Diese Katze stellt also gewissermassen den Grenzfall dar, bei dem in einzelnen Fällen Schaltung erfolgte, in anderen dagegen nicht.

1) Die Rückenmarke der Katzen von Versuch 3-7 wurden dem Zentralinstitut für Hirnuntersuchung in Amsterdam zur weiteren Bearbeitung übergeben. 
Zur Regelung der Bewegungen durch das Zentralnervensystem. III. 567

c) Zusammenfassung der Ergebnisse.

Auf die im Anfang dieses Abschnittes gestellte Frage nach der Abhängigkeit der geschilderten Schaltungsphänomene von den afferenten sensibelen Nerven haben, wie ich glaube, die im vorstehenden berichteten Versuche an Hunden und Katzen eine eindeutige Antwort geliefert. Nach Durchtrennung der hinteren Wurzeln ist die Lage und Stellung des Gliedes obne jeden Einfluss auf die Richtung der Reflexbewegungen, welche sich auf die verschiedenste Weise an diesen Gliedmaassen erhalten lassen. Dieses Ergebnis wird vielleicht manchem Leser als selbstverständlich erscheinen, ich glaube aber doch, dass es gut war, es über jeden Zweifel sicher zu stellen, weil nur auf dieser Basis eine weitere Analyse der Schaltungsphänomene möglich wird. In allen bisher geschilderten Versuchen wurde getrachtet, den Beweis zu liefern, dass die Motilität der untersuchten Gliedmaassen durch die Operation der Hinterwurzeldurchschneidung nicht beeinträchtigt worden ist. Erst wenn das asensibele Glied wieder nach allen Richtungen und mit genügender Energie bewegt werden konnte, wurde die Prüfung auf die An- oder Abwesenheit der Schaltungserscheinungen vorgenommen. Zugleich wurde der Sensibilitätsausfall genau bestimmt, um sicher zu sein, dass die beabsichtigte Operation auch wirklich ausgeführt worden war. Von den sensibelen Körperteilen liessen sich dann leicht kräftige Reflexbewegungen an den gefühllos gemachten Gliedern hervorrufen. Von den in den ersten beiden Mitteilungen dieser Reihe beschriebenen Gesetzmässigkeiten über die Richtung der Bewegung war aber in keinem Falle mehr etwas nachzuweisen.

\section{Durch welche zentripetalen Nerven wird die Schaltung vermittelt?}

Nachdem die im vorigen Abschnitte geschilderten Versuche ergeben hatten, dass die zu untersuchenden Schaltungen durch Vermittelung der sensibelen Bahnen in den Hinterwurzeln zustande kommen, war nun die weitere Aufgabe, zwischen den verschiedenen Arten afferenter Bahnen $z u$ scheiden und nachzusehen, welche von ihnen für das Zustandekommen des Phänomens entbehrlich und welche dafür notwendig sind. Der heutige Stand der experimentellen Technik erlaubt es, drei funktionelle Gruppen im Versuche von- 
einander zu sondern, die Sensibilität der Haut, der Gelenke und drittens die aller übrigen Strukturen zusammen, d. h. im wesentlichen der Muskeln selbst nebst den zugehörigen Sehnen und Faszien. Anfangs versuchte ich, die Trennung dieser Nervengruppen auf operativem Wege vorzunehmen, verliess diesen Weg aber bald wieder, weil z. B. die Durchtrennung aller sensibelen Hautnerven für den Oberschenkel und die angrenzenden Partien beim Hunde so ausgiebige Verletzungen nötig macht, dass das Glied nachher kaum noch als ein normales anzusehen ist, und man ausserdem wohl schwerlich sicher sein kann, nicht noch irgendeinen kleinen Nervenzweig undurchtrennt gelassen $z u$ haben. Viel besser eignet sich zu diesem Zwecke die Verwendung der modernen Lokalanästhetika, besonders seit der Einführung der neveren Kokainersatzmittel, welche wesentlich ungiftiger sind als das Kokain und besonders bei Zusatz von Suprarenin eine genügend langdauernde und starke Wirkung entfalten.

Die in diesem Abschnitt zu schildernden Versuche wurden ausscbliesslich an Rückenmarkshunden vorgenommen, bei denen die in der ersten Mitteilung beschriebenen Schaltungserscheinungen (Einfluss der Lage und Stellung des Gliedes auf die Richtung der Reflexbewegung bei gekreuzten Reflexen an den Hinterbeinen) geprüft wurden. Das Ergebnis war, dass nach Ausschaltung der Haut- und der Gelenksensibilität die Schaltungen noch mit genau derselben Sicherheit eintraten wie bei den Normalversuchen.

\section{Versuch 8.}

Hund „A“, 9 . Durchtrennung des Rückenmarkes in der Höhe des 12. Brustwirbels am 6. Mai 1909. Es ist dasselbe Tier, von welchem die in der I. Mitteilung wiedergegebenen kinematographischen Aufnahmen der Schaltungsversuche gewonnen wurden.

28. Juni 1909. Hund in guter Verfassung. Alle Umkehr- und Schaltungsreaktionen sind in deutlichster Weise zu erhalten. Bei Schlag auf die linke Kniesehne wird das rechte Bein, wenn es in allen Gelenken gebeugt gehalten wird, kräftig in allen Gelenken gestreckt, wenn es vorher aber in allen Gelenken gestreckt gehalten wird, ebenso kräftig gebeugt. Wird das in Knie- und Fussgelenk gestreckte Bein nur in der Hüfte gebeugt gehalten, so erfolgt Streckung des Hüftgelenkes. Bei Abduktion des Beines erfolgt als Reflexbewegung Adduktion, bei Adduktion dagegen Abduktion.

11 Uhr 34 Min. Injektion der drei grossen Gelenke des rechten Hinterbeines mit je $1 \mathrm{ccm} 5 \%$ igem Stovain unter Zusatz von 1/200 mg Suprarenin. 
Zur Regelung der Bewegungen durch das Zentralnervensystem. III. 569

Der Eingriff war vorher an der Leiche geübt worden. Die Injektion des Hüftgelenkes geschieht medial vom Beckenansatz des M. Adduktor longus, die Injektion ins Knie von der medialen, die ins Fussgelenk von der Vorderseite. Bei allen drei Gelenken ist das Eindringen der Injektionsnadel in die Gelenkhöhle gut wahrzunehmen, so dass man nicht im Zweifel bleibt, ob die Injektion auch wirklich ins Gelenk erfolgt ist.

Von $11 \mathrm{Uhr} 38 \mathrm{Min}$. bis $12 \mathrm{Uhr}$ werden die gekreuzten Reflexe vom linken auf das rechte Bein fortlaufend untersucht. Das rechte Bein hat dabei vorzügliche Motilität, alle Reflexe treten mit grosser Promptheit an demselben anf. Beim Aufrechthalten mit hängendem Hinterkörper pendeln beide Beine lebhaft, kommen aber dabei oft aus dem Takt und schlagen auch gelegentlich in verschiedener Frequenz. Das Pendeln lässt sich durch Unterstützen des Oberschenkels auf der rechten und linken Seite gleich gut hemmen. Passive Beugung und Streckung des rechten Beines löst starke Muskelreflexe am linken Hinterbeine aus. Der Patellarreflex ist auf der rechten Seite deutlich auszulösen, die Gegend des rechten Knies ist aber weniger empfindlich als die des linken.

Bei Schlag auf die linke Kniesehne erfolgen die oben geschilderten Umkehr- und Schaltungsreaktionen am rechten Beine genau in der gleichen Weise und mit derselben Sicherheit wie vor der Injektion der Gelenke. Daran ändert sich während der ganzen Dauer des Versuches nichts.

Bis zum Schluss des Versuches sind keine Erscheinungen von Allgemeinvergiftung des Tieres zu beobachten. Das Tier behält aber infolge der reizenden Eigenschaften des Stovains in den folgenden Wochen eine Beugekontraktur des Hüftgelenkes zurück. Daher wurde zu allen folgenden Versuchen das reizlose Novokain verwendet.

Resümee: Nach Anästhesierung der drei grossen Gelenke des rechten Hinterbeines am Rückenmarkshund bleiben alle Schaltungsreaktionen an diesem Beine unverändert bestehen.

Hund "Corry"

\section{Versuch 9.}

9. September 1909. Durchschneidung des Rückenmarkes am 10. Brustwirbel. Glatte Heilung. Die Reflexe entwickeln sich in vorzüglicher Weise. Die erste Schaltungsreaktion wird am 29. September beobachtet, in der Folge entwickeln sich alle in der I. Mitteilung beschriebenen Erscheinungen zu grösster Deutlichkeit. Vom 13. Oktober ab pendelt er mit den Hinterbeinen und zeigt auch die von Philippson beschriebenen Laufbewegungen, wenn man ihn mit horizontaler Wirbelsäule an Schultern und Schwanz frei in der Luft bält.

23. November. Bei einem Schlag auf die linke Kniesehne erfolgt am rechten Bein, wenn dasselbe in allen drei Gelenken gestreckt ist, Beugung der Hüfte (zugleich mit Beugung von Knie und Fuss), wenn dasselbe aber in der Hüfte gebeugt und in Knie und Fussgelenk gestreckt ist, Streckung der Hüfte. Es ist das der in der I. Mitteilung auf Fig. 2, 4 und 9 kinematographisch abebildete Versuch, in welchem nur durch veränderte Stellung in Hüftgelenk eine 
Schaltung zuwege gebracht wird, während Knie und Fussgelenk in beiden Fällen gleichmässig gestreckt bleiben. Dieser Versuch wird nun benutzt, um zu sehen, ob nach Anästhesierung der Haut des rechten Hüftgelenkes die Reaktion noch vorhanden ist. $\mathrm{Zu}$ diesem Zwecke wird die Haut des ganzen rechten Oberschenkels bis zum Knie und die Haut am Bauch, Rücken und Damm bis etwa in Nabelhöhe mit $150 \mathrm{~cm}$ einer $\mathbf{3} / \mathbf{4} \%$ igen Novokain-Suprareninlösung infiltriert. Darauf erfolgt prompte Anästhesie dieser Gegend. Die Grenze der asensiblen Zone beginnt in der Linea alba einen Finger breit unter dem Nabel, läuft $3 \mathrm{~cm}$ oberhalb der vierten Zitze gegen den Ansatz der Schenkelfalte, den sie in Nabelhöhe kreuzt, von da in derselben Höhe gegen die Wirbelsäule, längs dieser hinab bis zu After und Vulva, und am Bauche der Linea alba entlang bis kurz unterhalb des Nabels. Am Beine reicht die asensible Zone vorne und hinten bis zum Knie. Zur Feststellung der Hautsensibilität dienen die Auslösung des Kratzreflexes (vgl. die folgende Mitteilung) und an der Hinterseite des Oberschenkels und in der Sitzbeingegend Haarbewegungsreflexe, die bei diesem Tiere mit grosser Leichtigkeit erhalten werden können. - Der Ausfall der Sensibilität wird während der ganzen Dauer des Versuches kontrolliert und ändert sich in dieser Zeit nicht. Die Motilität des rechten Beines ist dabei ganz normal: Extensorstoss, Beugereflex, Patellarreflex, Kratzreflex lassen sich am rechten Beine mit Deutlichkeit auslösen. Beim Aufrechthängen erfolgt Pendeln der Hinterbeine nur, wenn man den Schwanz kneift. Bei Schlag auf die linke Kniesehne erfolgt, wenn das rechte Bein in allen Gelenkengestreckt ist, Beugung der Hüfte (manchmal begleitet von Beugung des Knies und Fusses); wenn dagegen das rechte Bein nur in Knie und Fussgelenk gestreckt, in der Hüfte dagegen gebeugt ist, erfolgt Streckung der Hüfte. Dieser Yersuch wird wiederholt angestellt und gelingt jedesmal mit Sicherheit und auf das deutlichste. - Gegen Schluss des Experimentes macht der Hund zweimal leichte Brechbewegungen. Die Beinreflexe sind alle gut zu erhalten, die Anästhesie besteht noch unverändert fort.

Resümee: Nach Anästhesierung der Haut in der ganzen Umgebung des rechten Hüftgelenkes am Rückenmarkshund bleibt die Schaltungsreaktion an diesem Hüftgelenke unverändert bestehen.

Derselbe Hund "Corry".

\section{Versuch 10 .}

23. Dezember 1909. Die Schaltungsreaktion am Hüftgelenk ist in derselben Weise auslösbar wie in Versuch 9. Von der Hant des l. Oberschenkels und der l. Bauchseite sind lebhafte Reflexe zu erhalten.

10 Uhr 35 Min. $1 \mathrm{ccm}$ 0,75\% iges Novokain wird zunächst in die Nähe des linken Hüftgelenkes, darauf $1 \mathrm{ccm}$ in das 1 . Hüftgelenk selber injiziert. Darauf sofort Infiltration der Haut auf der linken Seite ungefähr in derselben Ausdehnung wie beim vorigen Versuche (Verbrauch $90 \mathrm{~cm} 3 / 4 \%$ iges Novokain).

$10 \mathrm{Uhr} 50 \mathrm{Min}$. Asensibel ist der ganze linke Oberschenkel bis zum Knie, die ganze linke Bauchhaut bis zur Linea alba und bis einen Finger breit unterhalb des Nabels. Von hier geht die Grenze $2 \mathrm{~cm}$ unterhalb der drittletzten 
Zur Regelung der Bewegungen durch das Zentralnervensystem. III. 571

Zitze gegen den obersten Beginn der Schenkelfalte und gegen die Wirbelsäule, längs welcher sie nach hinten verläuft. Diese Sensibilitätsgrenze ändert sich im Verlaufe des Versuches nicht nachweisbar. Die Motilität des linken Beines ist gut. Beugereflex, Extensorstoss, Patellarreflex und Kratzreflex sird an ihm mit Deutlichkeit auszulösen. Deutliches Pendeln beider Beine.

11 Uhr. Bei Schlag a uf die rechteKniesehne erfolgt am linken Bein, wenn es inallen Gelenkengestrecktgehalten wird, Beugung von Hüfte, Knie undFuss, wenn das gestreckteBein dagegen nur in der Hüfte gebeugt wird, Streckung der Hüfte. Auch verschiedene andere Umkehrreaktionen lassen sich am l. Beine hervorrufen. Alles dieses wird wiederholt geprüft.

11 Uhr 15 Min. Das bis jetzt ruhige Tier bekommt einen deutlichen Erregungszustand mit Speichelsekretion und lebhafter Atmung. Aber auch jetzt bleibt die Schaltungsreaktion unverändert erhalten.

Resümee: Nach kombinierter Anästhesierung des Hüftgelenkes und der Haut in der ganzen Umgebung desselben bleibt am Rückenmarkshund die Schaltungsreaktion an diesem Gelenke unverändert bestehen.

Derselbe Hund "Corry".

\section{Versuch 11.}

27. Juni 1910. Alle Schaltungsreaktionen in deutlichster Weise auszulösen. Speziell erfolgt bei Schlag auf die linke Kniesehne, wenn das rechte Bein in allen Gelenken gebeugt ist, Streckung in allen Gelenken; wenn aber das rechte Bein in allen Gelenken gestreckt ist, Beugung in allen Gelenken. Wird das gestreckte Bein nur in der Hüfte gebengt, so erfolgt Streckung der Hüfte. Dieselben Reaktionen am rechten Beine werden auch erhalten, wenn statt des Schlages auf die linke Kniesehne als Reiz die schnelle Streckung des gebeugten linken Beines benutzt wird.

$10 \mathrm{Uhr} 25-30$ Min. Injektion des rechten Hüft-, Knie- und Fussgelenkes in der oben geschilderten Weise mit je $1 \mathrm{ccm}$ 0,75\% iger Novrkain-Suprareninlösung.

10 Uhr 40 Min. Infiltration der Haut des ganzen rechten Hinterbeines mit alleiniger Ausnahme der Zehenballen, sowie der Bauch-, Rücken- und Dammhaut in der gleichen Ausdehnung wie in den beiden vorhergehenden Versuchen (Verbrauch $115 \mathrm{ccm} 3 / 4 \%$ iger Novokain-Suprarenin). Danach wird die Haut des ganzen rechten Hinterbeines ausser den Zehenballen asensibel, ebenso die Bauch- und Rückenpartien bis zum vorderen Ansatz der rechten Schenkelfalte, sowie die rechte Hälfte des Dammes. Motilität des rechten Beines nicht gestört, Beugereflex, Extensorstoss, Patellarsehnenreflex und Kratzreflex sind an ihm deutlich zu erzielen.

10 Uhr 46 Min. Alle oben geschilderten Schaltungsreaktionen unverändert in deutlichster Weise demonstrierbar. Auf Schlagen der linken Kniesehne oder Strecken des gebeugten linken Beines erfolgt an dem infiltrierten rechten Beine, wenn dieses in allen Gelenken gebeugt ist, Streckung in allen Gelenken, wenn es in allen Gelenken gestreckt ist, Beugung in allen Gelenken. Wird das gestreckte rechte Bein nur in der Hüfte gebeugt gehalten 
so erfolgt Streckung der Hüfte. Alles dieses wird mehrfach geprüft. Die geschilderten Reaktionen treten konstant ein. Nach Șchluss der Versuche wird die Sensibilität nochmals kontrolliert und unverändert gefunden. Das Tier ist während des Versuches ganz ruhig. Nach Abschluss desselben entwickelt sich ein leichter Erregungszustand.

Resümee: Nach kombinierter Anästhesierung der drei grossen Gelenke des rechten Hinterbeines sowie der Haut an der rechten Seite von der Nabelgegend und dem oberen Ansatz der Schenkelfalte an abwärts bis zu den rechten Zehenballen bleiben am Rückenmarkshund alle Schaltungsreaktionen an diesem Beine unverändert bestehen.

\section{Zusammenfassung.}

Durch Versuche am Hinterbeine von Rückenmarkshunden wurde gezeigt, dass die in der I. Mitteilung beschriebenen Schaltungs- und Umkehrreaktionen nach Fortfall der Haut- und Gelenksensibiiität noch unverändert bestehen bleiben. Bei diesen Reaktionen wurde die Richtung der Reflexbewegung, welche auf einen kontralateralen Reiz eintrat, bestimmt durch die Lage und Stellung, welche dem Gliede vor Auflösung des Reflexes gegeben war. Einerlei, ob nur die Gelenke allein, oder ob nur die Haut allein, oder beide zusammen asensibel gemacht waren, einerlei ferner, ob nur die Reaktion des für diese Schaltungen wichtigsten Gelenkes, des Hüftgelenkes, oder ob die Reaktion des ganzen Beines mit allen drei Gelenken untersucht wurde, das Resultat war stets das gleiche: die Schaltungsreaktionen blieben unverändert erhalten.

Im Anfang dieser Arbeit wurde nun in einer Reihe von Versuchen an Hunden und Katzen gezeigt, dass die Schaltungsreaktionen endgültig aufgehoben werden, wenn alle sensiblen Nervenbahnen von dem betreffenden Gliede nach dem Zentralnervensystem durchgeschnitten worden sind. Daraus folgt, dass in den zuletzt geschilderten Experimenten die Schaltung im Zentralorgan bewirkt sein muss durch Vermittlung derjenigen sensiblen Bahnen, welche nach Ausschaltung der Haut und Gelenksensibilität noch von der betr. Extremität zum Rückenmark ziehen. Das können nur die afferenten Bahnen von den Muskeln (einschliesslich derer von den Sehnen und Faszien) sein.

In den ersten beiden Mitteilungen wurde ferner gezeigt, dass die bisher beschriebenen Fälle von Schaltung der von v. Uexkü ll bei Wirbellosen aufgestellten Regel folgen, nach welcher in einem diffusen Nervensystem eine Erregung immer mit besonderer Leichtig- 
Zur Regelung der Bewegungen durch das Zentralnervensystem. III. 573

keit den Zentren derjenigen Muskeln zufliesst, welche sich im Zustand der grössten Dehnung befinden. Auch bei unseren bisher beschriebenen Schaltungen floss im Rückenmark die Erreğung bei allen möglichen Reflexen mit besonderer Vorliebe den Zentren der gedehnten Muskeln zu.

Die in der vorliegenden Arbeit beschriebenen Experimente liefern nun, soweit wenigstens der Warmblüter in Frage kommt, die Erklärung für dieses Verbalten. Es handelt sich bei den besprochenen Schaltungen eben um Reflexe, welche von den Muskeln selber ausgelöst und auf dem Wege der sensiblen Muskelnerven dem Zentralorgan übermittelt werden ${ }^{1}$ ). Solange diese Bahnen funktionsfähig sind, so lange ist die Schaltung in den bisher beschriebenen Fällen möglich. Werden sie durchtrennt, so hört das geschilderte Phänomen auf.

\section{5. Über den zentralen Sitz der Schaltung.}

Schon in den früheren Mitteilungen war gezeigt worden, dass die Schaltungsphänomene nicht bedingt sein können durch die anatomische Anordnung der Skeletteile und der Muskeln, sondern dass sie im Zentralnervensystem lokalisiert werden müssen. Bei den Versuchen am Hundebein war diese Schlussfolgerung nicht einfach selbstverständlich gewesen, denn hier ist die anatomische Anordnung eine so verwickelte, dass sehr gut derselbe Muskel bei gebeugtem Beine eine Streckbewegung, bei gestrecktem eine Beugebewegung hätte hervorrufen können. Wenn trotzdem die Schaltung in das Zentralnervensystem lokalisiert wurde, so geschah es erstens deshalb, weil sich Bedingungen ergeben hatten, bei denen die anatomische Anordnung wohl dieselbe war, wo aber die Schaltungserscheinungen ehlten. Das war vor allem in der Zeit nach der Rückenmarksdurchschneidung der Fall gewesen, wenn die Shokfolgen allmählich zurückgingen, alle möglichen Beuge- und Streckreflexe wieder auftraten, ja selbst die Pendelbewegungen wieder begonnen hatten, aber noch keine Spur von einem Einfluss der Lage des Gliedes auf die Richtung der Reflexbewegungen sich nachweisen liess. Wenn dann einige Zeit später an demselben Tiere, ohne dass sich sein Muskeltonus und andere Bedingungen deutlich geändert hatten, die

1) Dabei bleibt zunächst unentschieden, ob die Schaltung bewirkt wird durch die Dehnung der einen Muskelgruppe oder durch die Entspannung ihrer Antagonisten oder durch beides zugleich. 
Schaltungen auf das deutlichste eintraten, so konnte dieses nicht gut auf etwas anderes als auf die veränderten Bedingungen des Zentralorganes, auf das Abklingen des Shoks zurückgeführt werden.

Derselbe Schluss auf die zentrale Lokalisation der Schaltungen ergab sich aus den Versuchen, in welchen das Bein als Ganzes immer gestreckt gelassen und nur die Stellung des Hüftgelenkes geändert wurde (vgl. z. B. oben Versuch 9 und die Kinematogramme der I. Mitt.). Hier handelte es sich nur um Bewegungen in einem Gelenke, und es war nicht einzusehen, wieso in diesem Falle das erhaltene Resultat auf die anatomische Anordnung der Gliedmaassen hätte bezogen werden können.

Drittens führten die Versuche am Katzenschwanz (II. Mitt.) zu demselben Ergebnis, denn an diesem einfach aus metameren Teilstücken aufgebauten Gebilde, das mit vier Gruppen von paarweise antagonistischen Muskeln versehen ist, wird das Resultat so ohne weiteres übersichtlich und anschaulich, dass wohl keiner die zentrale Natur des Phänomens bezweifeln dürfte.

$\mathrm{Zu}$ allem übrigen, um wieder auf die Bewegungen des Hundebeines zurückzukommen, hat nun in allerjüngster Zeit für die Beuge- und Streckbewegungen und für das Gehen und Laufen der Hinterbeine Sherrington ${ }^{1}$ ) eine ganz eingehende Analyse der mechanischen und nervösen Koordination durchgeführt, wobei er die Schaltungserscheinungen schon mit berücksichtigte. Dabei kam er zu dem Resultat, dass die Muskulatur der Gliedmaassen in ganz bestimmte funktionelle Gruppen zusammengeordnet ist, wovon die eine Gruppe immer nur bei der Streckung, die andere immer nur bei der Beugung in Kontraktion gerät. Nach Sherringtons Resultaten werden eben bei jeder Beugung, einerlei von welcher Ausgangsstellung aus sie erfolgt, immer nur die Beuger und niemals die Strecker kontrahiert und umgekehrt. Es kann also auch bei der Reflexumkehr infolge von Schaltung sich nicht darum handeln, dass eine Beugung des Beines durch Kontraktion der Streckmuskeln vorgetäuseht wird.

Nur für eine einzige Muskelgruppe ist Sherrington noch nicht zu völliger Klarheit darüber gelangt, ob sie sich diesem Gesetze durchaus fügt, das ist die Gruppe der Adduktoren. Schon

1) C. S. Sherrington, Flexion-reflex of the limb, crossed extension reflex and reflex stepping and standing. Journ. of Physiol. vol. 40 p. 28.1910. 
Zur Regelung der Bewegungen durch das Zentralnervensystem. III. 575

Lombard und $\mathrm{Abbott}{ }^{1}$ ) haben für das Froschbein auf eine solche Doppelfunktion der Adduktoren hingewiesen. Es war daher zu untersuchen, ob die Schaltungsreaktionen am Hundebein sich nach funktioneller Ausschaltung der Adduktorengruppe noch nachweisen lassen. Die Möglichkeit zu solchen Beobachtungen ergab sich an einem Hunde, bei welchem aus anderen Gründen beiderseits die Nerven der Adduktorengruppe, die Obturatorii durchtrennt waren.

\section{Versuch 12.}

Derselbe Hund ${ }_{n}$ Corry $^{*}$, welcher zu den Versuchen 9-11 gedient hat. Bei demselben hatten sich, wie in den früheren Protokollen erwähnt ist, nach der Anfang September 1909 vorgenommenen Querdurchtrennung des Rückenmarkes die Reflexe und alle Umkehrreaktionen in vorzüglicher Weise entwickelt. Das Tier war bis Ende Januar in Beobachtung gewesen und alle in der I. Mitteilung beschriebenen Versuche liessen sich an ihm mit grosser Deutlichkeit demonstrieren.

Am 26. Januar 1910 wurde in Morphinnarkose beiderseits im kleinen Becken neben der Vena hypogastrica der Nervus obturatorius durchschnitten und je ein Stück der Nerven exstirpiert. Bereits 2 Tage später liess sich an beiden Beinen vorzügliche Schaltung nachweisen. Bei Schlag auf die Kniesehne erfolgte am anderseitigen Bein, wenn es gestreckt war, Beugung, wenn es gebeugt war, Streckung.

Die Reflexumkehr durch Schaltung war also durch Ausschaltung der Adduktoren nicht aufgehoben, sondern sie war sogar deutlicher und übersichtlicher geworden, weil bei diesem Tiere seit der Operation immer nur reine Beugungen oder Streckungen erfolgten, welche nicht durch gleichzeitige Adduktionsbewegungen kompliziert waren. Es fielen also alle diejenigen Bewegungen fort, welche ich a. a. 0 . als Komplikationen beschrieben habe. Der Hund eignete sich deshalb in besonders guter Weise zur Demonstration der Schaltungsphänomene, und ich habe ihn seither oft zu diesem Zwecke benutzt.

Also auch nach Ausschaltung der Adduktorenbewegungen durch Nervendurchschneidung lässt sich der Einfluss der Stellung des Hundebeines auf die Richtung seiner Reflexbewegungen auf das deutlichste nachweisen. Es kann also anch eine etwaige Doppelfunktion dieser Muskejgruppe bei der Beugung und Streckung nicht für die Schaltungserscheinungen verantwortlich gemacht werden.

1) W. P. Lombard und Abbott, The mechanical effects produced by the contractions of individual muscles of the thigh of the frog. Americ. Journ. of Physiol. vol. 20 p. 1. 1907. 
Hält man das Resultat mit den erwähnten Ergebnissen Sherrington's zusammen, so ergibt sich auch hieraus der zentrale Angriffspunkt des Phänomens.

Alle diese etwas komplizierten Überlegungen werden aber nunmehr überflüssig, weil durch die in dieser Arbeit mitgeteilten Versuche der direkte Beweis erbracht wird, dass die hier geschilderten Schaltungen ihren Sitz im Rückenmark haben und nicht durch die anatomische Anordnung vorgetäuscht sind. Nach der Durchschneidung der hinteren Wurzeln hören alle Schaltungen, soweit sie bishęr untersucht wurden, auf. Die weiter oben ausführlich mitgeteilten Protokolle zeigen aber, dass sowohl die Hundebeine wie die Katzenschwänze, an denen diese Versuche angestellt wurden, eine vorzügliche Motilität besassen, dass sie nach allen Richtungen mit Kraft bewegt werden konnten, und in ihrer anatomischen Anordnung nicht gestört waren. Bei den Katzenschwänzen war ausserdem, wie das schon Merzbacher ${ }^{1}$ ausführlich für den Hund gezeigt, der Muskeltonus gar nicht herabgesetzt. Trotzdem war keine Spur von einem Einfluss der Stellung auf die Reflexrichtung mehr nachzuweisen. Wäre die Schaltung anatomisch in der Anordnung von Knochen und Muskeln bedingt, so hätte sie durch Hinterwurzeldurchschneidung nicht aufgehoben werden können.

Sämtliche in diesem Abschnitte angefuhrten Tatsachen führen also zu dem zwingenden Schlusse, dass die hier geschilderte Schaltung ein zentraler Vorgang ist, ausgelöst durch zentripetale Impulse, welche in den bisher studierten Fällen das Rückenmark auf dem Wege der sensiblen Muskelnerven erreichen.

\section{Die tonische Natur der Schaltung.}

Der Einfluss, den die Stellung und Lage des Gliedes anf die Richtung der nächstfolgenden Reflexbewegung dieses Gliedes ausübt, dauert in den hier untersuchten Fällen solange an, als das Glied sich in dieser Lage befindet. Davon kann man sich sowohl am Rückenmarkshund wie an der dezerebrierten Katze mit durchschnittenem Rückenmark überzeugen. Es macht keinen Unterschied,

1) L. Merzbacher, Die Folgen der Durchschneidung der sensiblen Wurzeln im unteren Lumbalmarke, im Sakralmarke und in der Cauda equina des Hundes. Pflüger's Arch. Bd. 92 S. 585. 1902. 
wenn man einen Rückenmarkshund auf den Rücken gelegt bat und ein Hinterbein in maximale Beugung oder Streckung bringt, ob man den reflexerzeugenden Reiz (Schlag auf die kontralaterale Kniesehne) sofort oder etwa nach 5 Minten anbringt. Die Richtung der Reflexbewegung ist in beiden Fällen mit derselben Sicherheit bestimmt.

Länger kann man die Versuche nicht gut ausdehnen, weil ein lebhafter Foxterrier meist nicht so lange in wirklich ruhiger Lage zu halten ist. Eine dezerebrierte Katze bleibt aber gewöhnlich in der ihr gegebenen Position liegen. Legt man eine Katze, welche zur Vornahme der Schaltungsversuche operiert ist, in Seitenlage auf den Tisch und lässt ibren Schwanz über den Tischrand nach unten hängen, so schlägt der Schwanz auf Berührung der Schwanzspitze immer nach oben, einerlei, ob man den Reiz sofort oder nach einer halben Stunde einwirken lässt. Der Einfluss dauert ebenso lange, als die Stellung und Lage des Gliedes andauert ${ }^{1}$ ).

Nun wurde in dem Vorgehenden gezeigt, dass die Schaltung eine reflektorische ist, die auf dem Wege der sensiblen Nerven im Zentralnervensystem zustande gebracht wird. Damit reiht sich das Phänomen also unter die Gruppe der tonischen Reflexe ein, und zwar bandelt es sich um tonische propriozeptive Reflexe, welche durch die afferenten Muskelnerven zustande gebracht werden. Nicht alle propriozeptiven Reflexe sind bekanntlich tonische. Sherrington hat eine ganze Reihe von Muskelreflexen beschrieben, welche im Gegenteil nur kurze Zeit andauern. Das sind die Reflexe, welche man am Räckenmarkstier durch passive Bewegung der Extremitäten auslösen kann. So bewirkt z. B. Streckung der Hüfte eine kontralaterale Beugung im anderen Hüftgelenk u. a. m. Diese Reflexe sind aber nur von kurzer Dauer und haben keinen tonischen Charakter. Tonische propriozeptive Reflexe treten dagegen mit Vorliebe an den Streckmuskeln dezerebrierter Tiere auf, wo sie zu der bekannten Enthirnungsstarre führen. Tonische Reflexe an Rückenmarkstieren sind viel seltener; ein von der Haut ausgelöster tonischer Beugereflex ist z. B. die Wochen und Monate andauernde Beugehaltung des Beines, wenn an der Haut sich irgend welche Ulzerationen entwickelt haben [Sherrington $\left.\left.{ }^{2}\right)\right]$.

1) Ebenso werden durch die rubige unveränderte Lage und Stellung eines Gliedes auch Dauer emp findungen (Lagegefühl) ausgelöst.

2) C. S. Sherrington, Flexion-reflex of the limb, crossed extension reflex and reflex stepping and standing. Journ. of physiol. vol. 40 p. 28.1910. 
Von allen diesen tonischen Reflexen unterscheiden sich aber die hier beschriebenen Erscheinungen dadurch, dass die afferenten Impulse selbst gar nicht den Eintritt irgendwelcher Bewegungen veranlassen (sie zum mindesten nicht veranlassen $m$ üssen). Die Einflüsse, um die es sich hier handelt, bewirken nur, dass ein nachfolgender Impuls eine bestimmte Bahn einschlägt und bestimmte Zentren in Erregung versetzt, andere Zentren, denen er auch zufliessen könnte, dagegen vermeidet. Es handelt sich eben nicht um Bewegungen oder Tonusänderungen der Muskulatur, sondern ausz schliesslich um „Schaltungen“ im Zentralorgan. Um ein naheliegendes Bild zu gebrauchen: es werden auf dem Rangierbahnhof nur die Wechsel gestellt, damit der nächste $\mathrm{Zug}$ richtig passieren kann. Es kann zweifelhaft erseheinen, ob für einen derartigen Vorgang noch der Ausdruck Reflex verwendet werden darf. Richtiger wäre es, nur von reflektorisch bedingter Schaltung zu sprechen. - Die Versuche zeigen, dass diese Vorgänge den Charakter von Dauerreaktionen haben.

\section{Die Rolle der Schaltung bei der Ausfïlırung normaler Körperbewegungen.}

Die bisherigen Auseinandersetzungen baben die Schaltung als einen wohlcharakterisierten und unter bestimmten Bedingungen mehr oder weniger konstant zu demonstrierenden zentralen Vorgang kennen gelehrt, und es erhebt sich nun die Frage, wie weit sich dieser Mechanismus bei der normalen Ausführung von Bewegungen beteiligt, wie weit er unter Umständen sogar dafür notwendig ist: Das Gehen stellt z. B. eine Bewegungsfolge dar, bei welcher antagonistische Muskelgruppen alternierend sich kontrahieren und erschlaffen. Nach den vorhergehenden Ausführungen sind in der Phase der grössten Beugung des Beines die Zentren der Streckmuskeln für alle möglichen Reize besonders leicht erregbar, in der Phase der grössten Streckung dagegen die Zentren der Beuger. Man wird hierdurch vor die Frage gestellt, ob etwa der Übergang von der Beugung zur Streckung oder der Übergang von der Streckung zur Beugung eine einfache Folge dieser Schaltung ist.

Sherring ton hat in seiner oben zitierten eingehenden Analyse der Beinbewegungen von Hunden und Katzen ${ }^{1}$ ) bereits diese

1) C. S. Sherrington, Flexion-reflex of the limb, crossed extension reflex and reflex stepping and standing. Journ. of Physiol. vol. 40 p. 28. 1910. 
Frage diskutiert und ist zu dem Schlusse gelangt, dass die Schaltung (Umkehr) nur als ein Hilfsmoment in Betracht kommen kann, und dass rein zentrale Vorgänge in Verbindung mit gleichseitigen und gekreuzten propriozeptiven Reflexen die alternierenden Gehbewegungen bedingen. Der Grund für diese Schlussfolgerung lag bauptsächlich darin, dass die Schaltung am Hundebeine die Bewegungsrichtung nicht zwangsmässig genug bedingt. In der ersten Mitteilung konnte ich zeigen, dass zwar beim gekreuzten Kniesehnenreflex die Bewegungsrichtung des Beines durch seine Lage und Stellung so gut wie eindeutig bestimmt wird, dass aber im Gegensatz dazu sich bei den gekreuzten Muskelreflexen, beim gekreuzten Extensorstoss u. a. diese Abhängigkeit nicht in allen Fällen nachweisen lässt. Gerade die Reflexe von den bewegten Muskeln sind aber nach Sherrington von besonders grosser Bedeutung für die Gehbewegungen.

Dass die Schaltung durch Lage und Stellung der Glieder keine völlig zwangsmässige ist, lehrt schon die einfache Selbstbeobachtung. Wir können willkürlich jederzeit ein gebeugtes Bein noch weiter beugen, ein gestrecktes noch weiter strecken. Bei der Rückenmarkskatze ist die Abbängigkeit der Bewegungsrichtung des Schwanzes von seiner Lage eine völlig zwangsmässige, und trotzdem kann eine normale nicht operierte Katze ihren nach links abduzierten Schwanz noch weiter nach links bewegen.

Die in dieser Mitteilung beschriebenen Versuche bestätigen die Folgerungen Sherrington's in jeder Hinsicht und erweitern sie. Der Hund "Gerda“ (Versuch 1), bei welchem das Rückenmark durchschnitten und die Hinterwurzeln für das eine Bein durchtrennt waren, führte mit diesem asensiblen Beine so gut wie mit dem normalen deutliche Pendelbewegungen aus, welche aus alternierenden Beugungen und Streckungen bestanden. Bei diesem Hunde waren aber alle Schaltungserscheinungen an dem asensiblen Beine völlig aufgehoben. Daraus folgt, dass die Schaltung keine notwendige Vorbedingung für das Zustandekommen alternierender Gehbewegungen ist. Dieselbe Folgerung muss man aus den Versuchen an Katzen mit asensiblem Schwanze ziehen. Auch bei ihnen waren alle Umkehrund Schaltungsreaktionen aufgehoben, und trotzdem konnten die Tiere während des Lebens noch rhythmisch alternierend mit dem Schwanze nach beiden Seiten schlagen.

Es können also die beschriebenen Schaltungen alternierende 
Bewegungen, wie das Gehen, Laufen, Schwanzwedeln usw., wohl unterstätzen und erleichtern, aber sie sind keine unerlässliche Vorbedingung für ihr Zustandekommen. Fs ist dieses wieder einer von den vielen Fällen, in denen eine wichtige Körperfunktion durch eine ganze Reihe verschiedener Einrichtungen „gesichert ${ }^{\text {}}$ ist. Im Falle der Gehbewegungen wirken ausser den zentralen Apparaten noch Reflexe von den bewegten Gliedmaassen selber und die hier beschriebenen Schaltungen zusammen.

Diese letzteren müssen ferner als unterstützende Sicherungen funktionieren bei allen Arten von Gleichgewichtsreaktionen des Körpers. Droht z. B. der Körper nach einer Seite umzufallen, so werden im allgemeinen die Muskeln der gegenüberstehenden Seite gedehnt werden. Setzt nun, um das Umfallen zu verhindern, irgendeine Gleichgewichtsreaktion ein, bei der sich diese gedehnten Muskeln kontrahieren, so findet dieser Reflex die Zentren der Muskeln, die er in Erregung versetzen muss, bereits in "eingeklinktem" Zustande vor. Es bedarf natürlich besonderer Untersuchungen, um festzustellen, in welchem Umfange nun tatsächlich der geschilderte Mechanismus bei den verschiedenen Gleichgewichtsreaktionen benutzt wird.

Es sei gestattet, an dieser Stelle noch einige Bemerkungen anzuschliessen, welche auf die praktische Medizin, und zwar auf die Orthopädie Bezug haben. Wenn, wie in dieser Arbeit gezeigt wurde, in vielen Fällen der Muskel selber je nach dem Grade seiner Dehnung auf die Erregbarkeit seines motorischen Zentrums im Rückenmarke einen Einfluss ausübt, so muss dadurch, dass man operativ einem Muskel einen anderen Ansatzpunkt am Skelette gibt, auch eine andere Erregbarkeitsänderung seines Zentrums bei Bewegungen dieses Skeletteiles erfolgen als vorher. Gibt man z. B. einem Ellbogenstrecker die Insertion eines Ellbogenbeugers, so wird der Muskel nunmehr im Gegensatz zu früher hei der Streckung des Gliedes gedehnt und bei der Beugung weniger gedehnt sein. Die Zentren dieses Muskels werden also bei Streckstellung „ein-“ und bei Beugestellung "ausgeklinkt" sein, und der Muskel, welcher vorher als Strecker funktionierte, muss nun, allein infolge seines veränderten Ansatzpunktes, mit besonderer Leichtigkeit als Beuger verwendet werden können.

Die hier angedeuteten Versuche sind in neuerer Zeit von den Orthopäden bei den verschiedensten Muskeln in zahlreichen Fällen und mit oft überraschend gutem und promptem Erfolge ausgeführt worden. Die Sehnenüberpflanzung hat sich bereits zu einer typischen Methode entwickelt. Ich entnehme der kurzlich erschienenen $\mathrm{zu}$ - 
Zur Regelung der Bewegungen durch das Zentralnervensystem. III. 581 sammenfassenden Darstellung von $\mathrm{Vulpius}{ }^{1}$ ) folgende Angaben:

Der Erfolg tritt auch ein, wenn statt eines funktionsverwandten Nachbarmuskels ein Antagonist verwendet wurde, ja, wenn statt der totalen Überpflanzung eine Funktionsteilung ausgeführt wurde. Dass nach der Sehnenüberpflanzung ein Muskel eine antagonistische Leistung übernehmen kann, darüber kann kein Zweifel bestehen. Codivilla sah nach Luxation der Peronealsehnen, dass die Muskeln als Extensoren des Fusses wirkten; sobald sie operativ in ihre normale Lage zurückgebracht waren, wurden sie ohne weiteres als Flexoren innerviert. Wird ein Muskel bzw. seine Sehne operativ der Länge nach gespalten, so kann die eine Hälfte die alte Funktion behalten, die andere die eines Antagonisten bekommen (Funktionsteilung). Solche Fälle sind am Tibialis anticus, am Extensor digitorum, am Wadenmuskel beobachtet. Am besten sieht man diesen Vorgang, wenn man einen Anteil der Trizepssehne auf den Biceps brachii überpflanzt. Hier lässt sich die isolierte Kontraktion der transplantierten Partie bei Flexion aufs deutlichste sehen und palpieren. In einem dieser Fälle begann das überpflanzte Trizepsstück sofort nach der Verbandabnahme in der neuen Weise zu funktionieren.

Diese Sätze lesen sich direkt wie eine experimentelle Bestätigung der oben entwickelten Vorstellungen. Ich glaube nun in der Tat, dass die Möglichkeit so weit gehender Sehnenüberpflanzungen mit gutem funktionellen Ergebnis auf Schaltungsvorgängen beruht, indem der Muskel in seiner neuen Lage unter anderen Verhältnissen arbeitet wie vorher, dementsprechend mit Hilfe seiner eigenen afferenten Nerven die Erregbarkeit seines motorischen Zentrums von Anfang an in der richtigen durch die neuen Verhältnisse geforderten Weise beeinflusst und so teils direkt seinem Zentrum im Rückenmark eine andere funktionelle Rolle aufzwingt, teils die Bedingungen schafft, durch welche im Verlaufe der Nachbehandlung das Grosshirn und die höheren Sinnesorgane diesen Mechanismus durch Übung verbessern können.

Sehr viel weniger einfach und übersichtlich liegen die Verhältnisse bei dem jüngsten Zweige der Lähmungsorthopädie, der Nervenüberpflanzung und Nervenpfropfung. Hier sind zweifellose günstige Resultate bekannt, bei denen eine Nervenbahn mit einem antagonistischen Muskel verbunden wurde und diesen dann in der funktionell richtigen Weise innervierte. $B$ eth $\mathrm{e}^{2}$ ) zitiert den Versuch von Flourens, der bei einem Huhne die beiden Haupt-

1) 0. Vulpius, Die Behandlung der spinalen Kinderlähmung. Leipzig 1910.

2) A. Bethe, Die Nervenregeneration und die Verheilung durchschnittener Nerven. Folia neurobiologica vol. 1 p. 63. 1907. 
stämme des Plexus brachialis kreuzweise miteinander durch die Naht vereinigte, und ein entsprechendes Experiment von $S$ te $p h$ a n $\mathrm{i}$ am Vorderbeine des Hundes. N. Tibialis und Peroneus wurden durch Forsmann und Spitzy ${ }^{1}$ ) beim Hunde kreuzweise miteinander vereinigt. Bethe machte dasselbe mit dem rechten und linken Ischiadicusstamm bei einem Hunde. In allen diesen Fällen war das funktionelle Resultat ein vorzügliches. Auch beim Menschen sind vor allem durch die Bemühungen Spitzy's ${ }^{2}$ ) schon gute Resultate erzielt worden. So lässt sich das gelähmte Radialisgebiet vom N. Medianus aus, das gelähmte Peroneusgebiet von N. Tibialis aus wieder frisch innervieren und wird dann in der funktionell richtigen Weise bei den Bewegungen benutzt. Nach den bisher vorliegenden Angaben der Chirurgen sind die Resultate aber keineswegs so sichere wie bei den Sehnenüberpflanzungen. Hierbei ist nun eins zu berücksichtigen. Wenn es richtig ist, dass die guten funktionellen Ergebnisse bei beiden Operationen auf Schaltungen beruhen, welche die Muskeln selbst auf dem Wege ihrer eigenen afferenten Bahnen im Rückenmark bewerkstelligen, so muss die Sehnenüberpflanzung schon an sich der Nervenplastik gegenüber im Vorteil sein, weil hier sicher die efferenten und die afferenten Nerven eines Muskelbauches beisammen bleiben. Bei der Nervenplastik wissen wir [Osborne und Kilvington $\left.{ }^{3}\right)$ ], dass die motorischen und sensiblen Bahnen des zentralen Stumpfes bei der Regeneration auch in andere periphere Bahnen hineingelangen können. Es bleibt daher dem Zufall und den speziellen anatomischen Bedingungen des Einzelfalles überlassen, ob nach der Plastik die afferenten Bahnen eines einzelnen Muskelbauches noch mit den zugehörigen motorischen Zentren in Verbindung stehen oder vielleicht sogar mit denen von antagonistischen Muskeln. Bei den zitierten Tierversuchen liegen die Verhältnisse, wie man sieht, besonders günstig, weil die kreuzweise verheilten Nerven zu funktionell sehr verschiedenartigen Gruppen gehören. Es ist anzunehmen, dass die Resultate der Nervenplastik sich noch

1) H. Spitzy, Weitere Erfahrungen auf dem Gebiete der Nervenplastik. Zeitschr. f. orth. Chir. Bd. 14 S. 671.1905.

2) H. Spitzy, Die Verwendung der Nervenplastik bei Plexuslähmungen. Zeitschr. f. orth. Chir. Bd. 16 S. 100. 1906.

3) W. A. Osborne und Kilvington, The arrangement of nerve fibres in a regenerated nerve trunk. Journ. of Physiol. vol. 38 p. 276. 1909. 
Zur Regelung der Bewegungen durch das Zentralnervensystem. III. 583 wesentlich verbessern werden, wenn bei der Operation darauf geachtet wird, dass nach der Regeneration die motorischen und sensiblen Babnen der verschiedenen Muskelbäuche beisammen bleiben.

\section{Schlusssätze.}

Die in früheren Mitteilungen beschriebenen Schaltungserscheinungen am Hundebein und Katzenschwanz, wobei die Stellung und Lage des Gliedes von Einfluss ist auf die Richtung einer nachfolgenden Reflexbewegung, sind nach Durchschneidung der hinteren Wurzeln nicht mehr zu beobachten.

Sie treten dagegen unverändert ein nach Ausschaltung der Haut- und Gelenksensibilität.

Die Schaltungen beruhen auf Vorgängen im Zentralnervensystem, die auf dem ${ }_{*}$ Wege der afferenten Muskel- (Sehnen-, Faszien-) Nerven von den Muskeln her ausgelöst werden.

Für die bisher beschriebenen Fälle gilt die v. Uexküll'sche Regel, wonach die Zentren der gedehnten Muskeln für eine Erregung anspruchsfähiger werden.

Der Einfluss der Lage und Stellung der Glieder auf die Erregbarkeitsverteilung in Zentralorgan ist ein dauernder (tonischer).

Es ist zu vermuten, dass die guten Erfolge der orthopädischen Sehnenüberpflanzungen und Nervenplastiken zum Teil auf den ge schilderten Schaltungserscheinungen beruhen. 